\title{
Exclusive chromomagnetism in heavy-to-light FCNCs
}

\author{
Maria Dimou, ${ }^{2, *}$ James Lyon, ${ }^{1,2, \dagger}$ and Roman Zwicky ${ }^{1,2, \dagger}$ \\ ${ }^{1}$ School of Physics and Astronomy, University of Edinburgh, Edinburgh EH9 3JZ, United Kingdom \\ ${ }^{2}$ School of Physics and Astronomy, University of Southampton, Highfield, Southampton SO17 1BJ, United Kingdom
}

(Received 17 December 2012; published 8 April 2013)

\begin{abstract}
We compute matrix elements of the chromomagnetic operator, often denoted by $\mathcal{O}_{8}$, between $B / D$ states and light mesons plus an off-shell photon by employing the method of light-cone sum rules at leading twist-2. These matrix elements are relevant for processes such as $B \rightarrow K^{*} l^{+} l^{-}$and they can be seen as the analogues of the well-known penguin form factors $T_{1,2,3}$ and $f_{T}$. We find a large $C P$-even phase for which we give a long-distance interpretation. We compare our results to QCD factorization for which the spectator photon emission is end-point divergent. The analytic structure of the correlation function used in our method admits a complex anomalous threshold on the physical sheet. The meaning and handling within the sum rule approach of the anomalous threshold is discussed.
\end{abstract}

DOI: 10.1103/PhysRevD.87.074008

PACS numbers: 12.38.- t, 13.25.Hw, 12.38.Lg

\section{INTRODUCTION}

Using the method of light-cone sum rules (LCSRs) (cf. Ref. [1] for a review), we present a computation of transition matrix elements

$$
\left\langle M(p) \gamma^{*}(q)\left|\mathcal{O}_{8}\right| H\left(p_{H}\right)\right\rangle, \quad p_{H}=p+q,
$$

of the chromomagnetic operator:

$\mathcal{O}_{8} \equiv-\frac{g}{8 \pi^{2}} m_{b} \bar{s} \sigma_{\mu \nu} G_{a}^{\mu \nu} \frac{\lambda^{a}}{2}\left(1+\gamma_{5}\right) b \equiv\left[-\frac{g m_{b}}{8 \pi^{2}}\right] \tilde{\mathcal{O}}_{8}$,

from the lowest lying meson $J^{P}=0^{-}$, denoted by $H$, with one heavy (beauty/charm) quark to a light pseudoscalar (vector) meson $M$ and a photon. Allowing the latter to be off shell leads to photon momentum invariant $q^{2}$ dependence of the matrix element. ${ }^{1}$ To our knowledge this work represents the first computation of the matrix elements (1). Factorizable parts have been computed in Refs. [2,3] to leading order in $1 / m_{b}$ and next leading order, though with end-point divergences [4,5], in QCD factorization (QCDF) as well as perturbative QCD (pQCD) [6]. For the $B \rightarrow \mathrm{Kl}^{+} l^{-}$transition the 3-particle $B$-meson state has been computed in LCSR recently [7].

We find that the matrix elements are suppressed by one (two) order(s) of magnitude for the $D(B)$ transitions with respect to the penguin short-distance (SD) form factors. Their interest is thus for asymmetries rather than for branching ratios. One example is the isospin asymmetry since the emission of the photon from the spectator quark is dependent on the charge of the decaying hadron; another observable is $C P$ violation, in combination with new weak

\footnotetext{
*md1e10@soton.ac.uk

†.D.Lyon@sms.ed.ac.uk

\#oman.Zwicky@ed.ac.uk

${ }^{1}$ We refrain from calling these matrix elements form factors since they entail long-distance (LD) contributions leading to a strong $(C P$-even $)$ phase.
}

phases, where the strong phase leads to direct $C P$ violation $[8,9]$. We shall also dwell on the nature of the end-point divergences found in QCDF and how they relate to LCSR results in which they are absent.

The paper is organized as follows: In Sec. II we define the matrix elements and present the basic sum rule including a brief discussion on anomalous thresholds and dispersion relations. In Sec. III the computation is presented including the final sum rule expression. In Sec. IV the numerics for the matrix elements are detailed as well as qualitative discussions. In Sec. V we compare our results with the QCDF computation in regard to end-point divergences. In Sec. VI we summarize the main points of the paper. Some explicit results and definitions can be found in Appendixes A, B, C, D, E, and F. Ward-Takahashi identities, clarifying the role of contact terms and the analytic structure of the correlation functions in use, can be found in Appendixes $\mathrm{G}$ and $\mathrm{H}$ respectively.

\section{MATRIX ELEMENT AND SUM RULE}

\section{A. Lorentz decomposition of $\tilde{\mathcal{O}}_{8}$ matrix elements}

For definiteness, throughout this work, we shall choose the initial state meson to be of the $\bar{B}$ type and the final state meson to be a vector meson $V$. Replacements of $B$ by $D$ mesons and vector $V$ by pseudoscalar $P$ are selfunderstood. The amplitude of the chromomagnetic operator, with uncontracted photon polarization vector $\epsilon(q)_{\rho}$, reads $^{2}$

$$
\begin{aligned}
\mathcal{A}^{* \rho}(V) & \equiv\left\langle\gamma^{*}(q, \rho) V(p, \eta)\left|\tilde{O}_{8}\right| \bar{B}\left(p_{B}\right)\right\rangle \\
& =i \int_{x}\left\langle V\left|T j_{\mathrm{em}}^{\rho}(x) \tilde{\mathcal{O}}_{8}(0)\right| \bar{B}\right\rangle e^{i q \cdot x}+\cdots .
\end{aligned}
$$

The dots stand for higher-twist photon distribution amplitude (DA) contributions, which are briefly discussed

\footnotetext{
${ }^{2}$ Note the right-hand side (rhs) of Eq. (3) should be taken as a definition of the matrix element $\mathcal{A}^{* \rho}(V)$ in the case where the photon is off shell.
} 
in Appendix B. The polarization vector of $V$ is denoted by $\eta$ and the momenta of $V, \gamma$ and $B$ are denoted $p, q$ and $p_{B} \equiv p+q$ respectively. Here and thereafter we use $\int_{x}=\int d^{4} x$. The star indicates that the photon is, generically, off shell. The operator $\tilde{\mathcal{O}}_{8} \equiv \bar{s} \sigma \cdot G\left(1+\gamma_{5}\right) b$ corresponds to $\mathcal{O}_{8}(2)$ without prefactors.

We define the dimensionless functions $G_{\iota}$, with $\iota \in$ $\{1,2,3, T\}$, as follows ${ }^{3}$ :

$$
\begin{aligned}
c_{V} \mathcal{A}^{* \rho}(V) & =k_{G}\left(G_{1}\left(q^{2}\right) P_{1}^{\rho}+G_{2}\left(q^{2}\right) P_{2}^{\rho}+G_{3}\left(q^{2}\right) P_{3}^{\rho}\right) \\
\mathcal{A}^{*}(P) & =k_{G}\left(G_{T}\left(q^{2}\right) P_{T}^{\rho}\right),
\end{aligned}
$$

with $k_{G}=-2 e / g$ to be explained further below. The transverse $\left(q_{\rho} P_{\iota}^{\rho}=0\right) \quad$ Lorentz structures $P_{i, T}, \quad$ of mass dimension $\left[P_{i}\right]=2$ and $\left[P_{T}\right]=1$, are given in Appendix E. The physical domain of the $B \rightarrow P(V) \gamma^{*} \rightarrow$ $P(V) l^{+}, l^{-}$transition is $\left(2 m_{l}\right)^{2} \leq q^{2} \leq\left(m_{B}-m_{P, V}\right)^{2}$, with $l$ being a lepton. ${ }^{4}$ Under exchange of chirality $\left(1+\gamma_{5}\right) \rightarrow\left(1-\gamma_{5}\right)$ in $\mathcal{O}_{8}(2)$, often denoted as $\mathcal{O}_{8}^{\prime}$, the $G_{\iota}$ functions transform as follows:

$$
\left\{G_{1}, G_{2}, G_{2}, G_{T}\right\}^{\left(1+\gamma_{5}\right) \rightarrow\left(1-\gamma_{5}\right)}\left\{G_{1},-G_{2},-G_{3}, G_{T}\right\},
$$

at leading order in the weak interactions. Thus $G_{1}$ and $G_{T}$ are parity conserving and $G_{2}$ and $G_{3}$ are parity violating. The operator $\mathcal{O}_{8}(2)$ is consistent with the effective Hamiltonian

$$
\begin{aligned}
\mathcal{H}_{\text {eff }} & =-\frac{G_{F}}{\sqrt{2}} V_{t s}^{*} V_{t b}\left(C_{7} \mathcal{O}_{7}+C_{8} \mathcal{O}_{8}\right)+\cdots, \\
\mathcal{O}_{7} & =-\frac{e m_{b}}{8 \pi^{2}} \bar{s} \sigma \cdot F\left(1+\gamma_{5}\right) b .
\end{aligned}
$$

In the case of $D \rightarrow M \gamma^{*}$ the replacements $b \rightarrow c, m_{b} \rightarrow$ $m_{c}$ and $V_{t s}^{*} V_{t b} \rightarrow V_{c b}^{*} V_{u b}$ are used. The normalization constant

$$
k_{G} \equiv-2 \frac{e}{g}
$$

used in (4) is chosen such that $G_{\iota}$ functions parallel the standard vector $T_{i}$ and pseudoscalar $f_{T}$ penguin form factors in the amplitude:

$$
\begin{aligned}
& \left\langle\gamma^{*}(q, \rho) V(p, \eta)\left|H_{\mathrm{eff}}\right| \bar{B}\right\rangle \\
& \quad \propto \sum_{i}\left(C_{7} T_{i}\left(q^{2}\right)+C_{8} G_{i}\left(q^{2}\right)\right) P_{i}^{\rho}+\cdots \\
& \left\langle\gamma^{*}(q, \rho) P(p)\left|H_{\mathrm{eff}}\right| \bar{B}\right\rangle \\
& \quad \propto\left(C_{7} f_{T}\left(q^{2}\right)+C_{8} G_{T}\left(q^{2}\right)\right) P_{T}^{\rho}+\cdots
\end{aligned}
$$

\footnotetext{
${ }^{3}$ The factor $c_{V}$ is inserted to absorb trivial factors due to the $\omega \sim(\bar{u} u+\bar{d} d) / \sqrt{2}$ and $\rho^{0} \sim(\bar{u} u-\bar{d} d) / \sqrt{2}$ wave functions. $c_{V}=-\sqrt{2}$ for $\rho$ in $b \rightarrow d$ transitions, $c_{V}=\sqrt{2}$ in all other transitions into $\omega$ and $\rho^{0}$ and $c_{V}=1$ otherwise.

${ }^{4}$ Analytic continuation to other values of $q^{2}$ can be related to other processes, e.g., $B+V \rightarrow \gamma^{*}$ by crossing symmetry. The domain of validity of our computation is discussed in Sec. IV.
}

\section{B. The sum rule}

The matrix elements (1) are extracted from the following correlation function ${ }^{5}$ :

$$
\begin{aligned}
\Pi^{V}\left(q^{2}, p_{B}^{2}\right) & =\epsilon^{* \rho}(q) \Pi_{\rho}^{V}\left(q^{2}, p_{B}^{2}\right) \\
& =i \int_{x}\left\langle\gamma^{*}(q) V(p)\left|T J_{B}(x) \tilde{O}_{8}(0)\right| 0\right\rangle e^{-i p_{B} \cdot x},
\end{aligned}
$$

where the $B$ meson figures as an interpolating current:

$$
J_{B}=i m_{b} \bar{b} \gamma_{5} q, \quad\left\langle\bar{B}\left(p_{B}\right)\left|J_{B}\right| 0\right\rangle=m_{B}^{2} f_{B} .
$$

In the equation above $q=u, d$ are light flavored quarks and $f_{B}$ is the standard $B$-meson decay constant.

Leaving aside the issue of parasitic cuts and how to compute the correlation function to the next section, we may apply standard techniques of dispersion relations and Borel transformations [10] to extract the matrix element under consideration. The dispersion representation of the correlation function in the variable $p_{B}^{2}$,

$$
\Pi^{V}\left(q^{2}, p_{B}^{2}\right)=\frac{1}{2 \pi i} \oint_{\bar{\Gamma}} \frac{d s \Pi^{V}\left(q^{2}, s\right)}{s-p_{B}^{2}},
$$

is nothing but Cauchy's integral theorem: the closed path $\bar{\Gamma}$ is chosen such that no singularities, including anomalous thresholds (to be discussed in the next section), are crossed. An example is shown in Fig. 1 for the analytic structure of the correlation function in QCD; $\bar{\Gamma}=\bar{\Gamma}_{\mathrm{P}} \cup \bar{\Gamma}_{\mathrm{C}}$. In a second step advantage is taken of the isolated $B$ pole by splitting the dispersion integral into two parts as follows:

$$
\begin{aligned}
\Pi^{V}\left(q^{2}, p_{B}^{2}\right)= & \frac{m_{B}^{2} f_{B}}{m_{B}^{2}-p_{B}^{2}}\left\langle\gamma^{*}(q) V(p)\left|\tilde{O}_{8}\right| \bar{B}\left(p_{B}\right)\right\rangle \\
& +\frac{1}{2 \pi i} \oint_{\bar{\Gamma}_{\mathrm{C}}} \frac{d s \Pi^{V}\left(q^{2}, s\right)}{s-p_{B}^{2}} .
\end{aligned}
$$

Equating (11) and (12) one obtains

$$
\begin{aligned}
& \frac{m_{B}^{2} f_{B}}{m_{B}^{2}-p_{B}^{2}}\left\langle\gamma^{*}(q) V(p)\left|\tilde{O}_{8}\right| \bar{B}\left(p_{B}\right)\right\rangle \\
& \quad=\frac{1}{2 \pi i}\left(\oint_{\bar{\Gamma}} \frac{d s \Pi^{V}\left(q^{2}, s\right)}{s-p_{B}^{2}}-\oint_{\bar{\Gamma}_{\mathrm{C}}} \frac{d s \Pi^{V}\left(q^{2}, s\right)}{s-p_{B}^{2}}\right) .
\end{aligned}
$$

For the purpose of numerical improvement a Borel transformation,

$$
B_{s \rightarrow M^{2}}\left[\frac{1}{x-s}\right]=\frac{e^{-x / M^{2}}}{M^{2}},
$$

in the variable $p_{B}^{2}$ is applied to (13) to obtain

$$
\left\langle\gamma^{*}(q) V(p)\left|\tilde{O}_{8}\right| \bar{B}\left(p_{B}\right)\right\rangle=D\left[\Pi^{V}, \bar{\Gamma}\right]-D\left[\Pi^{V}, \bar{\Gamma}_{\mathrm{C}}\right]
$$

\footnotetext{
${ }^{5}$ For the sake of notational simplicity we shall keep the photon polarization tensor contracted here as with respect to (3), though from a physical point of view this does not make sense for an offshell photon.

${ }^{6}$ Possible subtraction terms, due to ultraviolet (UV) divergences, are ignored in view of the fact that they disappear under Borel transformation.
} 

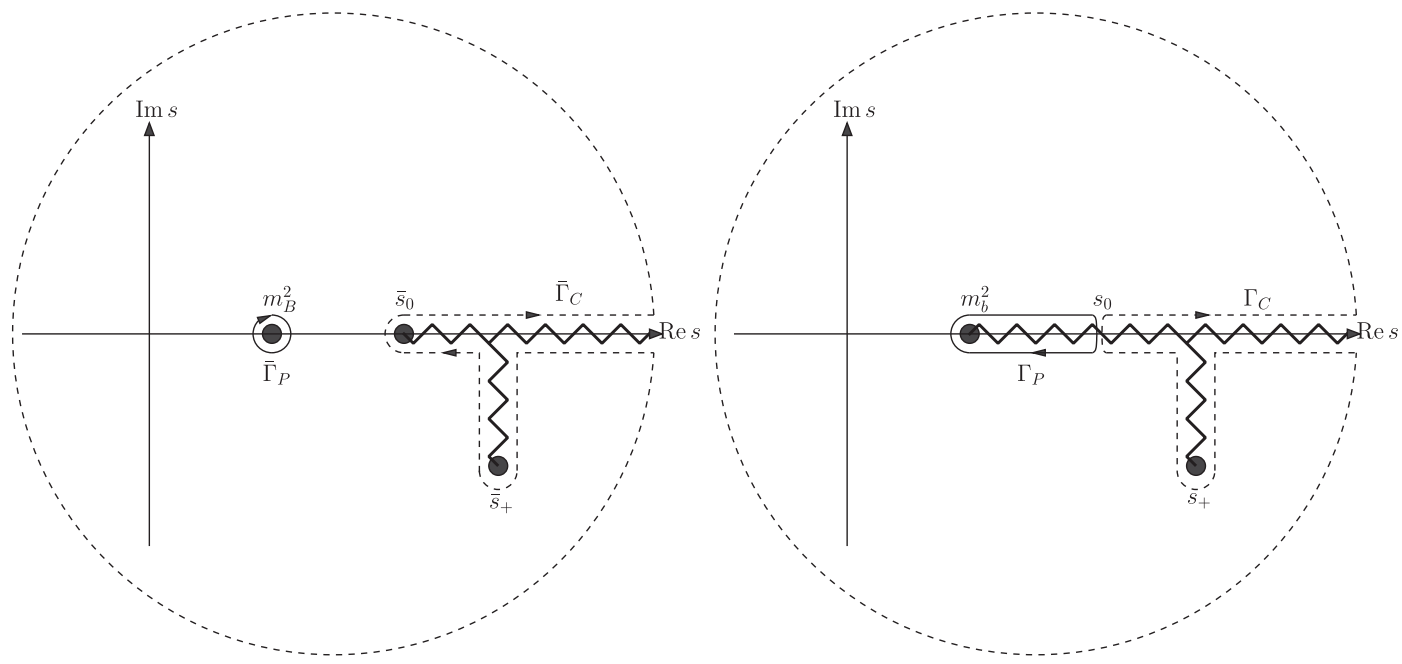

FIG. 1. $\Gamma_{\mathrm{P}}\left[\bar{\Gamma}_{\mathrm{P}}\right]$ and $\Gamma_{\mathrm{C}}\left[\bar{\Gamma}_{\mathrm{C}}\right]$ correspond to the straight and dashed paths in the right (left) figure respectively. (left) Analytic structure of the correlation function in QCD. There is an isolated $B$ pole at $s=m_{B}^{2}$ and a branch point $\bar{s}_{0}=\left(m_{B}+2 m_{\pi}\right)^{2}$ at the continuum threshold. Furthermore the existence of a complex branch point $\bar{s}_{+}$, which corresponds to an anomalous threshold, can be inferred from the work of Källén and Wightman; cf. Appendix H 1 c. The path $\bar{\Gamma}=\bar{\Gamma}_{P} \cup \bar{\Gamma}_{\mathrm{C}}$ is a possible path for Eq. (11). (right) Analytic structure of the correlation function as found in leading order perturbation theory. The branch point related to the normal threshold starts at $m_{b}^{2}$. The existence of the anomalous branch point $s_{+}$is shown in Appendixes $\mathrm{H} 1 \mathrm{a}$ and $\mathrm{H} 1 \mathrm{~b}$ respectively. The two branch points $\bar{s}_{+}$and $s_{+}$ are expected to be close, but not identical, in the same way as $m_{B}^{2}$ is close to $m_{b}^{2}$.

where we introduce the shorthand notation

$$
D\left[f, \Gamma_{f}\right] \equiv \frac{1}{f_{B} m_{B}^{2}} \frac{1}{2 \pi i} \oint_{\Gamma_{f}} d s e^{\left(m_{B}^{2}-s\right) / M^{2}} f\left(q^{2}, s\right) .
$$

The expression in (15), up to neglecting the width of the $B$ meson, is exact although rather cryptic. Approximations enter the calculation of the correlation function $\Pi^{V}$ due to neglecting higher twist and $\alpha_{s}$ corrections and in estimating $D\left[\Pi^{V}, \bar{\Gamma}_{\mathrm{C}}\right]$. Let us be more precise about the latter point. Whereas $D\left[\Pi^{V}, \Gamma\right] \approx D\left[\left.\Pi^{V}\right|_{\mathrm{LC}-\mathrm{OPE}}, \bar{\Gamma}\right]$ is a good approximation for off-shell $p_{B}^{2}$ up to the truncations in twist and $\alpha_{s}$ mentioned above, the approximation $D\left[\Pi^{V}, \bar{\Gamma}_{\mathrm{C}}\right] \approx D\left[\left.\Pi^{V}\right|_{\mathrm{LC}-\mathrm{OPE}}, \Gamma_{\mathrm{C}}\right]$, which goes under the name of semiglobal quark hadron duality, is less transparent and usually the main limitation of a sum rule computation. In the full theory $\bar{\Gamma}_{\mathrm{C}}$ marks the onset of the continuum threshold which corresponds to the lowest lying multiparticle state [e.g., $\bar{s}_{0}=\left(m_{B}+2 m_{\pi}\right)^{2}$ in QCD]. ${ }^{7}$ For the light-cone operator product expansion (LC-OPE) dispersion representation one introduces an effective continuum threshold $s_{0}[1,10],{ }^{8}$ which corresponds to the duality approximation mentioned above.

\footnotetext{
${ }^{7}$ In principle there might be further isolated states, with $B\left(0^{-}\right)$ quantum numbers, between $m_{B}^{2}$ and $\left(m_{B}+2 m_{\pi}\right)^{2}$. Note that there are none listed in PDG [11]. In our discussion those states would simply be included into the path $\bar{\Gamma}_{\mathrm{C}}$.

${ }^{8}$ Whereas $s_{0} \approx \bar{s}_{0}$ ought to be the case exactness cannot be expected to hold. Realistically one can expect $s_{0}$ to be somewhere between say $\left(m_{B}+2 m_{\pi}\right)^{2}$ and $\left(m_{B}+m_{\rho}\right)^{2}$. Whether or not this affects the final result depends on the convergence of the LC-OPE and Borel parameters. This effect has to be analyzed which is done in Sec. IV.
}

The crucial point in connection with the anomalous threshold, which results in branch cuts extending into the complex plane, is that its real part is above the continuum threshold, $m_{b}^{2}+m_{B}^{2} / 2>s_{0}$, and therefore it is entirely included in $\Gamma_{C}$ and will not contribute to the final sum rule. ${ }^{9}$ Therefore the path $\Gamma$ minus the path $\Gamma_{\mathrm{C}}$ corresponds to the path $\Gamma_{\mathrm{P}}$ that encircles the real line segment from $m_{b}^{2}$ to $s_{0}$. The final sum rule can be written as

$$
\begin{aligned}
& \left\langle\gamma^{*}(q) V(p)\left|\tilde{O}_{8}\right| \bar{B}\left(p_{B}\right)\right\rangle \\
& \simeq D\left[\left.\Pi^{V}\right|_{\mathrm{LC}-\mathrm{OPE}}, \Gamma\right]-D\left[\Pi_{\mathrm{LC}-\mathrm{OPE}}^{V}, \Gamma_{\mathrm{C}}\right] \\
& =D\left[\left.\Pi^{V}\right|_{\mathrm{LC}-\mathrm{OPE}}, \Gamma_{P}\right] \\
& =\frac{1}{f_{B} m_{B}^{2}} \int_{m_{b}^{2}}^{s_{0}} d s e^{\left(m_{B}^{2}-s\right) / M^{2}} \rho^{V}\left(q^{2}, s\right)
\end{aligned}
$$

with

$$
\begin{aligned}
2 \pi i \rho^{V}\left(q^{2}, s\right) & =\operatorname{Disc}_{s} \Pi^{V}\left(q^{2}, s\right) \\
& =\Pi^{V}\left(q^{2}, s+i 0\right)-\Pi^{V}\left(q^{2}, s-i 0\right),
\end{aligned}
$$

where we have dropped the subscript LC-OPE in (18). Note the radius of the path $\Gamma_{\mathrm{C}}$ and $\Gamma$ (as well as for the barred quantities) does not enter the final relation (17). The important point is that the end point of the duality interval is much larger than the intrinsic scale of QCD; $s_{0} \gg \Lambda_{\mathrm{QCD}}^{2}$.

\footnotetext{
${ }^{9}$ It is also suppressed by the Borel transformation, both due to the large real part of $s$ and the oscillation in the exponential due to $\operatorname{Im} s \neq 0$ along the associated branch cut.
} 


\section{Remarks on dispersion relations and anomalous thresholds}

As the appearance of complex singularities in forms of anomalous thresholds is rather nonstandard in sum rule computations, we consider it worthwhile to add a few remarks. Our three main points are

(i) We note, again, that Eq. (11) is nothing but the application of Cauchy's integral theorem. Thus knowledge of the analytic structure of the correlation function is mandatory.

(ii) The existence of the pole at the $B$-meson mass ${ }^{10}$ and its residue in terms of the matrix elements in Eq. (12) can be inferred from derivations like the one presented in Chap. 10.2 in Ref. [12].

(iii) The part not related to the $B$-meson pole, i.e., the part encircled by $\Gamma_{\mathrm{C}}$, is to the rhs of $\operatorname{Re}\left[s_{0}\right]$. In practice this means that it is suppressed, by the Borel transformation (14), by at least a factor of $e^{\left(m_{B}^{2}-s_{0}\right) / M^{2}}$ with respect to the $B$-pole part.

A few remarks on the connection between physical states and singularities: For a two-point function, ${ }^{11}$ a dispersion representation is in one-to-one correspondence with the insertion of a complete set of states as is explicit in the celebrated Källén-Lehmann representation [13] and derivations thereof. Thus, the analytic structure, in the complex plane of the four-momentum invariant, has a cut and poles on the real line starting from the lowest state in the spectrum. For correlation functions with three or more fields, there is no such direct relation. The analytic structure can be more involved as singularities other than those related to intermediate states might appear, known as anomalous thresholds, e.g., Refs. [14,15]. Singularities related to unitarity, that is to say to an insertion of a complete set of states, are called normal thresholds. From the viewpoint of a dispersion relation, normal and anomalous thresholds should be viewed as being on the same footing, ${ }^{12}$ as only the analytic structure counts. Which singularities are relevant for the physics in question is another matter. Clearly, here we are interested in the matrix element corresponding to the residue of the pole of the $B$ meson which belongs to the normal part. The arguments above should make it clear that the anomalous thresholds do no more harm than any other continuum contribution to the extraction of the matrix element in question.

\footnotetext{
${ }^{10}$ Ignoring the finite width, which otherwise moves the pole into the lower half-plane of the second Riemann sheet.

${ }^{11}$ In this paragraph it is assumed that the operators are gauge invariant.

${ }^{12}$ Let us add that even among the normal thresholds there are states which do not correspond to the insertion of a single identity. e.g., the parasitic states which correspond to different time ordering. As discussed in this paper they do appear when no momentum is flowing into one of the operators of the correlation function.
}

\section{THE COMPUTATION}

In this section we provide more details of the computation with some explicit results deferred to the Appendixes. At leading order in $\alpha_{s}$ there are a total of 12 graphs. They can be split into those where the gluon connects to the spectator $(s)$ and the ones where it connects to the nonspectator (ns) quark:

$$
G_{\iota}\left(q^{2}\right)=G_{\iota}^{(s)}\left(q^{2}\right)+G_{\iota}^{(\mathrm{ns})}\left(q^{2}\right) .
$$

The four diagrams denoted by $A_{1}$ to $A_{4}$ in Fig. 2 (top, middle) contribute to $G_{\iota}^{(s)}$ whereas the diagrams at the bottom of the same figure correspond to the $G_{\iota}^{(\mathrm{ns})}$ contributions. Hereafter we use $\bar{u} \equiv 1-u$. The $G_{\iota}^{(\text {ns) }}$ functions factorize into a function $f\left(q^{2} / m_{b}^{2}\right)$ times the standard vector, axial or tensor form factors. The function $f$ has been obtained in the inclusive case in Ref. [16], ${ }^{13}$ in terms of an expansion in powers of $q^{2} / m_{b}^{2}$ and logarithmic terms. The two diagrams where the gluon connects to the nonspectator quark and photon emission from the latter are not shown. These diagrams are expected to be small, since no fraction of the $m_{b}$ rest mass is transmitted to the energetic photon and we shall neglect them. For the same reason and for being of higher twist we expect the diagrams where the gluon is radiated into the final state meson to be suppressed. ${ }^{14}$

\section{A. The problem of parasitic cuts}

Because of the fact that there is no momentum flowing into the weak vertex at $\tilde{\mathcal{O}}_{8}$, there is an ambiguity in separating the cuts corresponding to the $B$ meson from other cuts. The general problem originates from the fact that the relation between correlation functions of higher degree and matrix elements is complicated by time ordering and a nontrivial analytic structure. Similar issues appear in Euclidean field theory and represent an obstacle to extracting a matrix of more than two hadronic states from correlation functions on the lattice [19]. In LCSR the problem is best understood by first introducing its (partial) cure.

We follow the method introduced by Khodjamirian for $B \rightarrow \pi \pi$ [20], which might be seen as an extension of earlier ideas [21], and introduce a spurious momentum $k$ into the weak vertex. This introduces two further momenta denoted by $P=p_{B}-k$ and $Q=q-k$. Formally, the $1 \rightarrow 2$ decay is augmented by the spurious momentum $k$ to a $2 \rightarrow 2$ scattering process which has six independent kinematic variables: $\left\{q^{2}, Q^{2}, p_{B}^{2}, P^{2}, k^{2}, p^{2}\right\}$. Without any consequence for our purposes we can set $q^{2}=Q^{2}$ and $k^{2}=0$. Capital $Q$ will from now on only be used for the

\footnotetext{
${ }^{13}$ We would like to add that it would be possible to compute these contribution within LCSR itself.

${ }^{14} \mathrm{~A}$ rough estimate can be given by comparing the similar case where a gluon is radiated from a charm loop, instead of $\mathcal{O}_{8}$ to the hard spectator or the final state meson. Taking the estimates of Refs. [2,17,18] we find roughly a factor of 4 between them.
} 

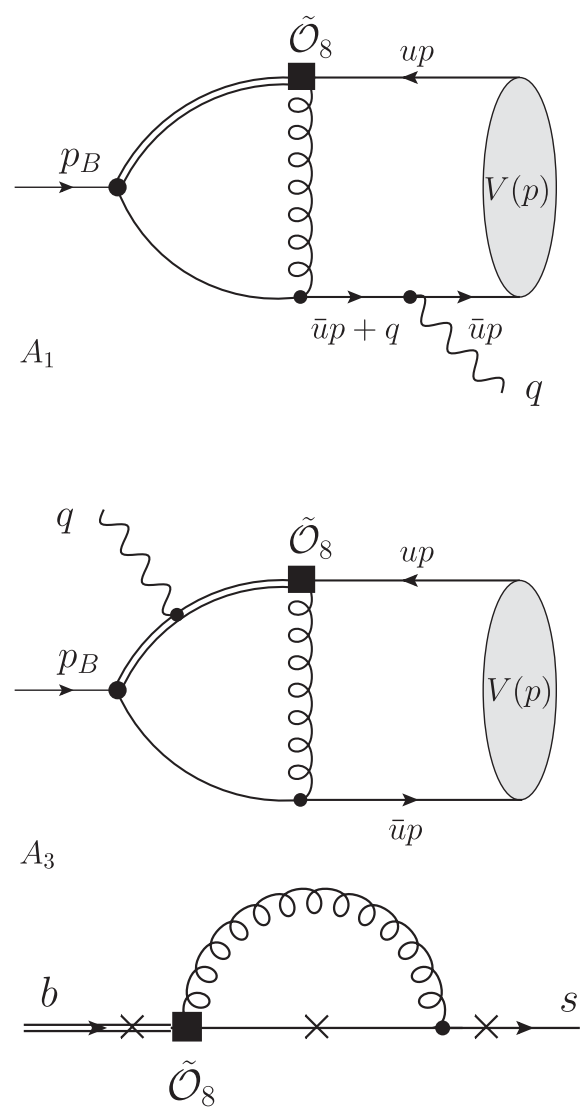
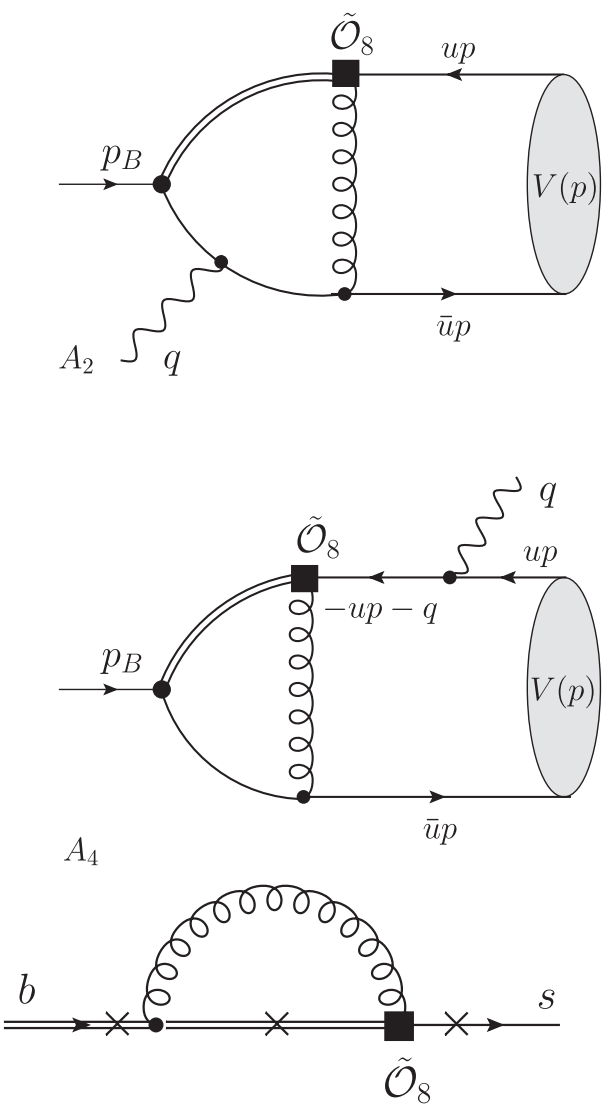

FIG. 2. (top/middle) Diagrams $A_{1}$ to $A_{4}$ correspond to all four possibilities with the gluon from the weak vertex connecting to the spectator quark. (bottom) Nonspectator corrections. They have been computed in Ref. [16] and factorize into a form factor and $B \rightarrow V / P$ form factor as described in Appendix $\mathrm{D}$. The crosses indicate all possible photon insertions.

four-momentum throughout the paper. Recalling that $p^{2}=m_{P, V}^{2}$ the six kinematical invariants are reduced to $\left\{q^{2}, P^{2}, p_{B}^{2}\right\}$ which we shall discuss in the next section. The variable $P^{2}$ remains the only trace of the spurious momentum at this stage. How it effectively disappears from the final result is discussed in the next subsection after we discuss the lightlike dominance of the correlation function. At the level of the correlation function (9) the change is implemented by changing the photon momentum $q \rightarrow Q$. The above mentioned cuts then branch into cuts in $p_{B}^{2}$ and $P^{2}$ (cf. Fig. 3), where the former correspond to the $B$ meson and the latter to parasitic ones.

The extension of the Lorentz structures to the case where we include the spurious momentum $k$ is given in Appendix E 1. Using the latter we parametrize the correlation functions as follows:

$$
\begin{aligned}
& \Pi^{V}=\sum_{i=0}^{4} g_{i}\left(q^{2}\right) \epsilon(Q) \cdot p_{i}, \\
& \Pi^{P}=\sum_{i \in\{0, T, \bar{T}\}} g_{i}\left(q^{2}\right) \epsilon(Q) \cdot p_{i},
\end{aligned}
$$

where $\epsilon(Q)$ is the photon polarization tensor and $\left(p_{0}\right)_{\rho}=$ $Q_{\rho}$ is a nontransverse structure related to contact terms.
As previously stated the Lorentz structures corresponding to the $G_{\iota}$ functions are transverse even for an offshell photon. This is not necessarily true for the correlation function. Why these terms are there and why they do not affect the extraction of the matrix element is discussed in Appendix $G$ in terms of a Ward-Takahashi identity (WTI).

\section{B. The light-cone expansion}

The correlation function is expected to be dominated by lightlike distances in the case where the kinematical invariants $k^{2}, q^{2}, p_{B}^{2}$ and $P^{215}$ are below the thresholds. In that case, LC-OPE (cf. Ref. [1] for a review on the topic) is applicable. For the physical matrix element $q^{2}$ and $P^{2}$ necessitate analytic continuation, an issue which we defer to Secs. III C and IV B respectively. Schematically the LC-OPE reads as follows:

\footnotetext{
${ }^{15}$ The remaining two invariants are $Q^{2}=q^{2}$ and $p^{2}=m_{P, V}^{2}$. The former thus does not necessitate a separate statement and the latter is on shell by virtue of being the momentum of a physical state.
} 


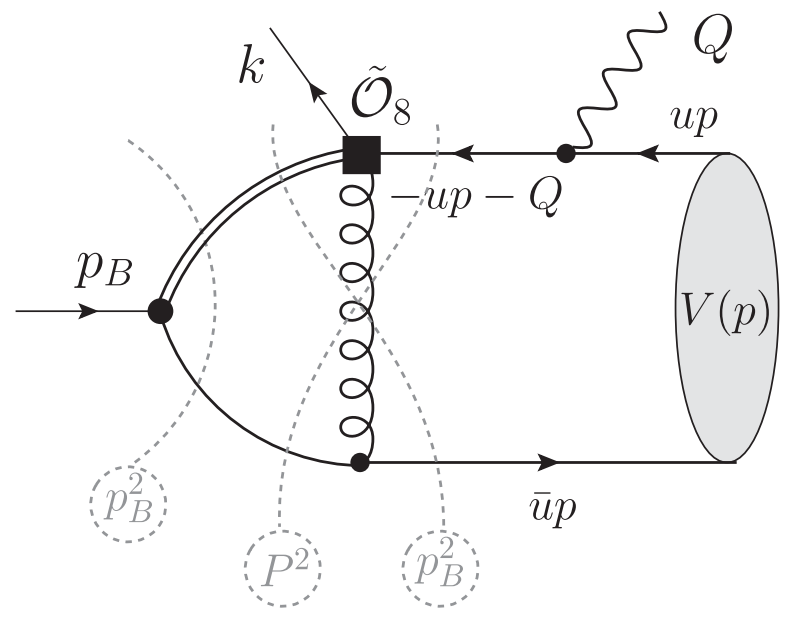

FIG. 3. Various cuts in the variables $p_{B}^{2}$ and $P^{2} \equiv\left(p_{B}-k\right)^{2}$. The cut in $P^{2}$ is of a parasitic type in the sense that for $k \rightarrow 0$ it cannot be distinguished from $p_{B}^{2}$ yet it is clearly not associated with the $B$ meson as it does not cut in the $b$-quark line. The two cuts in $p_{B}^{2}$ are of the 2-parton and 3-parton type and both should be (and are) included. Here and thereafter the double line denotes the $b$-quark propagator.

$$
\Pi\left(q^{2}, p_{B}^{2}, P^{2}\right)=\sum_{i} T_{H}^{(i)}\left(q^{2}, p_{B}^{2}, P^{2} ; \mu_{F} ; u\right) \circ \phi^{(i)}\left(u, \mu_{F}\right),
$$

where $i$ sums over different distribution amplitudes (DAs) of increasing twist. The twist corresponds to the dimension of the operator minus its spin. The terms are suppressed by $\Lambda_{\mathrm{QCD}}$ over the virtuality to the power of the twist. In this work we limit ourselves to the leading twist-2. The relevant DAs are summarized in Appendix F. The variable $u$ represents generic parton momentum fractions, the symbol $\circ$ stands for the integration over the latter and $T_{H}$ is a perturbatively calculable hard kernel. The symbol $\mu_{F}$ denotes the collinear factorization scale and separates, within the LCOPE, the SD physics in the kernel $T_{H}$ from the LD part in the DA. This scale should not be confused with the renormalization scale $\mu_{\mathrm{UV}}$ to be discussed in the numerics section. For the computation we use FeynCalc [22]. We would like to highlight two issues in connection with the calculation:

(i) Infrared (IR) divergences. - We note that the diagram $A_{2}$ in Fig. 2 has a potential soft divergence for $p^{2} \rightarrow 0$ and a collinear divergence for $q^{2} \rightarrow 0$. The former cancels and the latter appears only in the $P_{3}$ and $P_{T}$ Lorentz structures which do not contribute at $q^{2}=0$.

(ii) Schouten identity.-For structures like $Q_{\rho} \epsilon\left(\eta, p, p_{B}, Q\right)$ the Schouten identity $g^{a b} \epsilon^{\text {cdef }}=$ $g^{a c} \epsilon^{b d e f}-g^{a d} \epsilon^{b c e f}-g^{a e} \epsilon^{b d c f}-g^{a f} \epsilon^{b d e f}$ has to be used since they contain pieces of the Lorentz structure $\left(p_{1}\right)_{\rho}$ in (E3).

UV divergences are present in diagrams $A_{2}$ and $A_{3}$ but are of no consequence as the discontinuities of the correlation functions do not depend on them. Explicit results in terms of Passarino-Veltman (PV) functions [23] and their corresponding dispersion relations, including the handling of the complex branch cuts, are given in Appendixes A, B, C, D, E, F, G, and $\mathrm{H}$ respectively.

\section{Analytic continuation and appearance of strong phases}

As previously stated the LC-OPE is valid when all invariants take on values such that no thresholds are crossed. To obtain a physical result two of those invariants, $q^{2}$ and $P^{2}$, need to be analytically continued: $q^{2}$ to enter the physical domain for $B \rightarrow V(P) l l$ transitions and $P^{2}$ to eliminate the spurious momentum $k$.

For $B \rightarrow V(P) l l$ the physical range for $q^{2}$ is between $\left(2 m_{l}\right)^{2}$ and $\left(m_{B}-m_{P, V}\right)^{2}$ and it has become customary to exclude the region below $1 \mathrm{GeV}^{2}$ in order to avoid the $(\rho, \omega)$-resonance region. For $B \rightarrow V \gamma$, which corresponds to $q^{2}=0$, it can be argued that one is still considerably low. ${ }^{16}$ More details concerning individual graphs and the high $q^{2}$ region can be found in Sec. IV B. As previously stated, the only trace of the spurious momentum is in $P^{2} \equiv\left(p_{B}-k\right)^{2} \neq p_{B}^{2}$. This trace can be lifted by analytically continuing $P^{2} \rightarrow m_{B}^{2}+i 0$. Note that if we had the full solution of the correlation function, then $p_{B}^{2}=m_{B}^{2}$ would lead to an exact projection by virtue of a LehmannSymanzik-Zimmermann reduction. In the sum rule approximation the remnant of this is the fact that the integral representation (17) averages over a narrow range of $m_{B}^{2}$. On the level of the LC-OPE, this analytic continuation is expected to hold as it is far above all thresholds; the variable $P^{2}$ does not cut through the $b$-quark line (cf. Fig. 3). Both analytic continuations lead to LD contributions which in turn lead to strong phases. This is illustrated for a $P^{2}=m_{B}^{2}$ cut in Fig. 4 (left) and for a $q^{2} \simeq m_{\rho}^{2}$ cut in Fig. 4 (right).

In summary, both $q^{2}$ and $P^{2}$ are analytically continued sufficiently far above the thresholds, much like the open charm region in $e^{+} e^{-} \rightarrow(\bar{c} c) \rightarrow e^{+} e^{-}$.

\section{RESULTS, SUMMARY AND NUMERICS}

We note that in the sum rule the product $\left[m_{B}^{2} f_{B}\right] \times$ $\left\langle\gamma^{*}(q) V(p)\left|\tilde{O}_{8}\right| \bar{B}\left(p_{B}\right)\right\rangle$ [cf. Eq. (12)], rather than the $G_{i}\left(q^{2}\right)$ functions themselves are extracted. This suggests that one should use a sum rule determination of the same order in the quantity $\left[m_{B}^{2} f_{B}\right]^{17}$ in order to extract the matrix element(s). Such a strategy has for example been proposed in Ref. [24]. From Fig. 3 it is evident that the 2-particle cut corresponds to a decay constant of order $\mathcal{O}\left(\alpha_{s}^{0}\right)$. The 3 -particle cut in the same figure corresponds partially to an $\mathcal{O}\left(\alpha_{s}\right)$ correction. We expect the former to be dominant so we feel justified to use the sum rule result to order $\mathcal{O}\left(\alpha_{s}^{0}\right)$,

\footnotetext{
${ }^{16} q^{2}=0$ is sufficiently below the $(\rho, \omega)$ threshold region and therefore the LC-OPE is expected to work.

${ }^{17}$ This quantity corresponds to the matrix element of the interpolating current (10).
} 

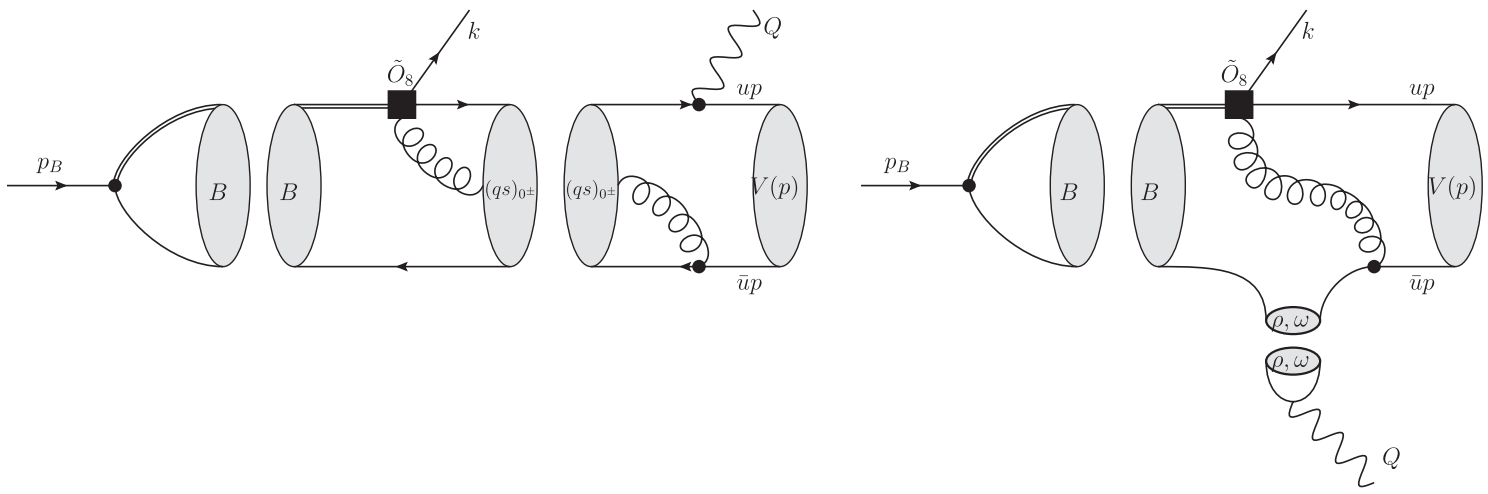

FIG. 4. (left) Hadronic interpretation of the 3-particle cut in Fig. 3 in terms of a LD hadronic process. The latter is a source for the strong $\left(C P\right.$-even) phase that we obtain for the $G_{\iota}\left(q^{2}\right)$ functions. (right) Hadronic interpretation of the strong phase due to $q^{2}>0$, associated with $B \rightarrow V(\rho, \omega) \rightarrow V \gamma^{*} \rightarrow V l l$ type transitions.

$$
\begin{aligned}
{\left.\left[m_{B_{q}}^{2} f_{B_{q}}\right]^{2}\right|_{\mathrm{SR}_{0}}=} & \left(m_{b}+m_{q}\right)^{2} e^{\frac{m_{B}^{2}-m_{b}^{2}}{M^{2}}}\left(-m_{b}\langle\bar{q} q\rangle_{\mu}-\frac{m_{b}}{2 M^{2}}\left(1-\frac{m_{b}^{2}}{2 M^{2}}\right)\langle\bar{q} G q\rangle_{\mu}\right. \\
& \left.+\frac{3}{8 \pi^{2}} \int_{\left(m_{b}+m_{q}\right)^{2}}^{s_{0}} e^{\frac{m_{b}^{2}-s}{M^{2}}}\left(s-\left(m_{b}-m_{q}\right)^{2}\right) \sqrt{\left(s-m_{b}^{2}-m_{q}^{2}\right)^{2}-4 m_{b}^{2} m_{q}^{2}} \frac{d s}{s}\right)
\end{aligned}
$$

which has been known for a long time [25]. The parameters $M^{2}=M^{2}\left[f_{H_{q}}\right]$ and $s_{0}=s_{0}\left[f_{H_{q}}\right]$ are not, necessarily, the same as the ones in the sum rule for $G_{\iota}$ functions. Further discussion is deferred to Appendix $\mathrm{C}$.

Following the decomposition (19) at twist-2 the spectator parts decompose for the vector and pseudoscalar final state as follows:

$G_{i}^{(s)}=G_{i}^{(\perp)}\left(q^{2}\right)+G_{i}^{(\mathrm{ll})}\left(q^{2}\right), \quad G_{T}^{(s)}=G_{T}^{(P)}\left(q^{2}\right)$.

The superscripts $\{\perp, \|, P\}$ refer to the projections onto the corresponding light-meson DA, e.g., (F1). ${ }^{18}$ For the sake of clarity it would be better to replace the notation by $\left.G_{i}^{(\perp)} \rightarrow G_{i}\right|_{\phi_{\perp}}$ but we shall not do so in order to retain a compact notation. Out of the seven functions (23), four satisfy relations so that the full function can be reconstructed by three of them:

$$
V: G_{1}^{(\perp)}\left(q^{2}\right), G_{3}^{(\|)}\left(q^{2}\right), \quad P: G_{T}^{(P)}\left(q^{2}\right) .
$$

The four relations required are $G_{1}^{(\mathrm{II})}\left(q^{2}\right)=0, G_{2}^{(\mathrm{Il})}\left(q^{2}\right)=0$, $G_{2}^{(\perp)}=\left(1-q^{2} / m_{B}^{2}\right) G_{3}^{(\perp)}$ and $G_{2}^{(\perp)}=\left(1-q^{2} / m_{B}^{2}\right) G_{1}^{(\perp)}$. The third relation assures a finite decay width in the limit $m_{V}^{2} \rightarrow 0$ (as employed here); cf. Appendix A and Ref. [26]. The fourth relation is of the large energy effective theory (LEET)-type as found for the form factors in Ref. [27]. The latter can be explained at this level in a straightforward way cf. Appendix A. Furthermore, in the ultrarelativistic approximation $m_{V}^{2} \rightarrow 0$, the projections $G_{T}^{(P)}\left(q^{2}\right)$ and $G_{3}^{(I I)}\left(q^{2}\right)$

\footnotetext{
${ }^{18}$ Note that these labels are not necessarily in one-to-one correspondence with the amplitudes $\mathcal{T}_{\perp, \|, P}$ as used in Ref. [3].
}

are proportional to each other modulo a replacement of the corresponding DA; cf. Appendix A.

For the sake of completeness, we shall give the sum rule expression for $G_{1}^{(\perp)}\left(q^{2}\right)$

$$
\begin{aligned}
G_{1}^{(\perp)}\left(q^{2}\right) & =\frac{1}{\left.\left(m_{B}^{2} f_{B}\right)\right|_{\mathrm{SR}_{0}}} \int_{m_{b}^{2}}^{s_{0}} e^{\frac{m_{B}^{2}-s}{M^{2}}} \rho_{1}^{(\perp)}(s) \\
\rho_{1}^{(\perp)}(s) & =p \int_{0}^{1} d u \phi_{\perp}(u) \sum_{i=a}^{d}\left(b_{i}^{\perp} \rho_{B_{i}}(u, s)+c_{i}^{\perp} \rho_{C_{i}}(u, s)\right),
\end{aligned}
$$

where $p \equiv C_{F}\left(\alpha_{s} / 4 \pi\right) f_{V}^{\perp} m_{b}^{2} Q_{b} /(-2)$, the sum runs from $a$ to $d$ alphabetically and $\rho_{B(C)_{i}}$ and $b(c)_{i}^{\perp}$ are given in Eqs. (H4) and (A11) respectively.

The central hadronic input parameters and their uncertainties are given in Appendix $\mathrm{C}$. The collinear factorization scale is chosen to be $\mu_{F}^{2}=m_{b}\left(m_{c}\right) \Lambda_{\text {had }} \simeq$ $m_{b}\left(m_{c}\right) 0.8 \mathrm{GeV}$ for $B(D)$ transitions. This scale corresponds to the momentum transfer and is standard for hard-spectator contributions. We consider all types of FCNC $b \rightarrow(d, s), c \rightarrow u$ transitions of a $B(D)$ meson into a light $V(P)$ meson as indicated in Table I, with the exception of $P=\eta, \eta^{\prime}$. This sums up to a total of 19 transitions: 11 to a vector and 8 to a pseudoscalar. Central values at $q^{2}=0$ for $G_{1}^{(\perp)}(0)$, as required for $B(D) \rightarrow V \gamma$ transitions (cf. Sec. IV C), and uncertainties are collected in Table II. In Appendix $\mathrm{C}$ values for all transitions for $q^{2}=0 \ldots m_{b(c)}^{2}$ are collected in Table IV. The validity of the $q^{2}$ range of our computations is discussed in Sec. IV B. 
TABLE I. FCNC transitions up to charge conjugation for $B(D) \rightarrow V(P)$ as indicated. The valence quark content of the mesons is indicated in brackets and the type of transition is indicated. We do not consider $\eta$ and $\eta^{\prime}$ for the pseudoscalars. There are a total of $11_{V}+8_{P}=19$ transitions.

\begin{tabular}{lcccccccc}
\hline \hline & $\begin{array}{c}\rho[\pi]^{+} \\
(u \bar{d})\end{array}$ & $\begin{array}{c}\rho[\pi]^{0}, \omega \\
(\bar{u} u) \pm(\bar{d} d)\end{array}$ & $\begin{array}{c}\rho[\pi]^{-} \\
(u \bar{d})\end{array}$ & $\begin{array}{c}K^{*}[K]^{+} \\
(u \bar{s})\end{array}$ & $\begin{array}{c}K^{*}[K]^{0} \\
(d \bar{s})\end{array}$ & $\begin{array}{c}K^{*}[K]^{-} \\
(s \bar{u})\end{array}$ & $\begin{array}{c}\bar{K}^{*}[\bar{K}]^{0} \\
(s \bar{d})\end{array}$ & $\begin{array}{c}\phi \\
(s \bar{s})\end{array}$ \\
\hline$B^{-}$ & $(b \bar{u})$ & & & $b \rightarrow d$ & & & $b \rightarrow s$ & \\
$\bar{B}^{0}$ & $(b \bar{d})$ & & $b \rightarrow d$ & & & & & $b \rightarrow s$ \\
$\bar{B}_{s}$ & $(b \bar{s})$ & & & & & & & \\
$D^{0}$ & $(c \bar{u})$ & & $c \rightarrow u$ & & & & & \\
$D^{+}$ & $(c \bar{d})$ & $c \rightarrow u$ & & & $c \rightarrow u$ & & & \\
$D_{s}$ & $(c \bar{s})$ & & & & $c \rightarrow u$ & & \\
\hline \hline
\end{tabular}

TABLE II. The contribution of the diagrams $A_{1}-A_{4}$ of Fig. 2 at $q^{2}=0$ for an on-shell photon. One observes that on a qualitative level there are four types of transitions, the $B$ or $D$ and charged or uncharged. The notation $(b D)^{0}$ for instance means a $b \rightarrow(d, s)$ transition in a charge neutral meson. In all cases, the charge conjugate transition follows by simply reversing the sign, since all amplitudes are proportional to the charges of the valence quarks. Together with the nonspectator correction $G_{i}^{(n s)}$, this constitutes the relevant information for $B(D) \rightarrow V \gamma$ decays. Note $G_{1}^{(\perp)}(0)=G_{2}^{(\perp)}(0)$. Further information is given in Sec. IV C. The uncertainties in the real and imaginary parts are very close and we thus refrain from quoting them separately.

\begin{tabular}{|c|c|c|c|c|c|c|c|}
\hline & $G_{1}^{(\perp)}(0) \cdot 10^{2}$ & Uncertainty \% & Type & & $G_{1}^{(\perp)}(0) \cdot 10^{2}$ & Uncertainty $\%$ & Type \\
\hline$B^{-} \rightarrow \rho^{-} \gamma$ & $0.29-0.39 i$ & 25 & $(b D)^{-}$ & $\bar{B}_{s} \rightarrow K^{* 0} \gamma$ & $0.21+0.18 i$ & 27 & $(b D)^{0}$ \\
\hline$B^{-} \rightarrow K^{*-} \gamma$ & $0.29-0.40 i$ & 26 & $(b D)^{-}$ & $\bar{B}_{s} \rightarrow \phi \gamma$ & $0.26+0.23 i$ & 26 & $(b D)^{0}$ \\
\hline $\bar{B}^{0} \rightarrow \rho^{0} \gamma$ & $0.22+0.19 i$ & 27 & $(b D)^{0}$ & $D^{0} \rightarrow \rho^{0} \gamma$ & $-7.0-5.0 i$ & 32 & $(c u)^{0}$ \\
\hline $\bar{B}^{0} \rightarrow \omega \gamma$ & $0.19+0.17 i$ & 33 & $(b D)^{0}$ & $D^{0} \rightarrow \omega \gamma$ & $-6.1-4.3 i$ & 34 & $(c u)^{0}$ \\
\hline $\bar{B}^{0} \rightarrow \bar{K}^{* 0} \gamma$ & $0.20+0.20 i$ & 28 & $(b D)^{0}$ & $\begin{array}{l}D^{+} \rightarrow \rho^{+} \gamma \\
D^{+} \rightarrow K^{*+} \gamma\end{array}$ & $\begin{array}{l}-1.9+2.5 i \\
-1.8+2.1 i\end{array}$ & $\begin{array}{l}32 \\
33\end{array}$ & $\begin{array}{l}(c u)^{+} \\
(c u)^{+}\end{array}$ \\
\hline
\end{tabular}

Let us turn to the discussion of uncertainties. We vary the Borel parameters $M^{2}[G], M^{2}\left[f_{H}\right]$, the continuum threshold $s_{0}$, the heavy quark mass $m_{b}$, the decay constants and the condensates as indicated in Appendix C. The major uncertainties come from varying $s_{0}, m_{h}$ and $\mu_{F}$ which amount to about $11 \%$ [15\%], $8 \%[7 \%]$, and $5 \%$ [20]\% for $B[D]$ transitions respectively. The uncertainties in the decay constants can be significant depending on the final state meson as they enter linearly. We expect violation of quark-hadron duality to be accounted for by variations of $s_{0}$. There are two further sources of uncertainty which are not taken care of by varying parameters. First, the scale dependence of the operator $\tilde{O}_{8}\left(\mu_{\mathrm{UV}}\right),{ }^{19}$ especially since we do not include proper radiative corrections in $\alpha s$. At 1-loop level the diagonal anomalous dimension is $\gamma_{88}=C_{F}$ in conventions where $\gamma_{m}=6 C_{F}$ and is fortunately small. Evolving at leading $\log$ level from $\mu=1 \mathrm{GeV}$ to $m_{b}$ leads to a $7 \%$ effect which we shall adapt as an estimate of this uncertainty. Second, the omission of twist- 3 and higher twists: on grounds of past experience we attribute a $15 \%$ uncertainty to them. Note that the Borel mass is chosen to suppress the latter, yet keeping violations of quark-hadron duality acceptably

\footnotetext{
${ }^{19}$ In physical processes, such as $B \rightarrow K^{*} \gamma$, this is compensated by the Wilson coefficients. The leading order Wilson coefficient $\gamma_{88}$ would enter our computation only if we were to go to one more order in $\alpha_{s}$.
}

small, as explained in Appendix C. Finally all the parametric variations, as described above, and the uncertainty of higher twist and $\mu_{\mathrm{UV}}$ are added in quadrature, as we do not see a reason for strong correlations. The final uncertainties along the central values are collected in Table II.

\section{A. Qualitative discussion}

As discussed in the caption of Table II there are four qualitatively different transitions depending on whether the initial meson is either of $b$ or $c$ flavor and on whether it is charged or not, which is of course a manifestation of the sensitivity to isospin. The $b$ types are plotted in Fig. 5. The $q^{2}$ dependence is somewhat more complex than the one of an ordinary form factor $B \rightarrow \pi$. In the latter case the $q^{2}$ dependence is merely governed by a series of poles, starting at $q^{2}=m_{B^{*}}^{2}$, and higher multihadron cuts. For this reason fitting that form factor is rather simple. In our case at hand, as discussed in the next subsection, the photon couples to all kinds of flavors and thus poles in $q^{2}=m_{\rho}^{2}, m_{B^{*}}^{2}, \Upsilon(\bar{b} b)$ appear. Furthermore there are genuine LD contributions which result in strong phases for $q^{2}$, $P^{2}>0$ as discussed in Sec. III C and illustrated in Fig. 4. Moreover we note that the imaginary part decreases with $q^{2}$. This is to be expected as the process shown in Fig. 4 (left) is more and more off shell for higher $q^{2}$, at least at leading order $\alpha_{s}$. 

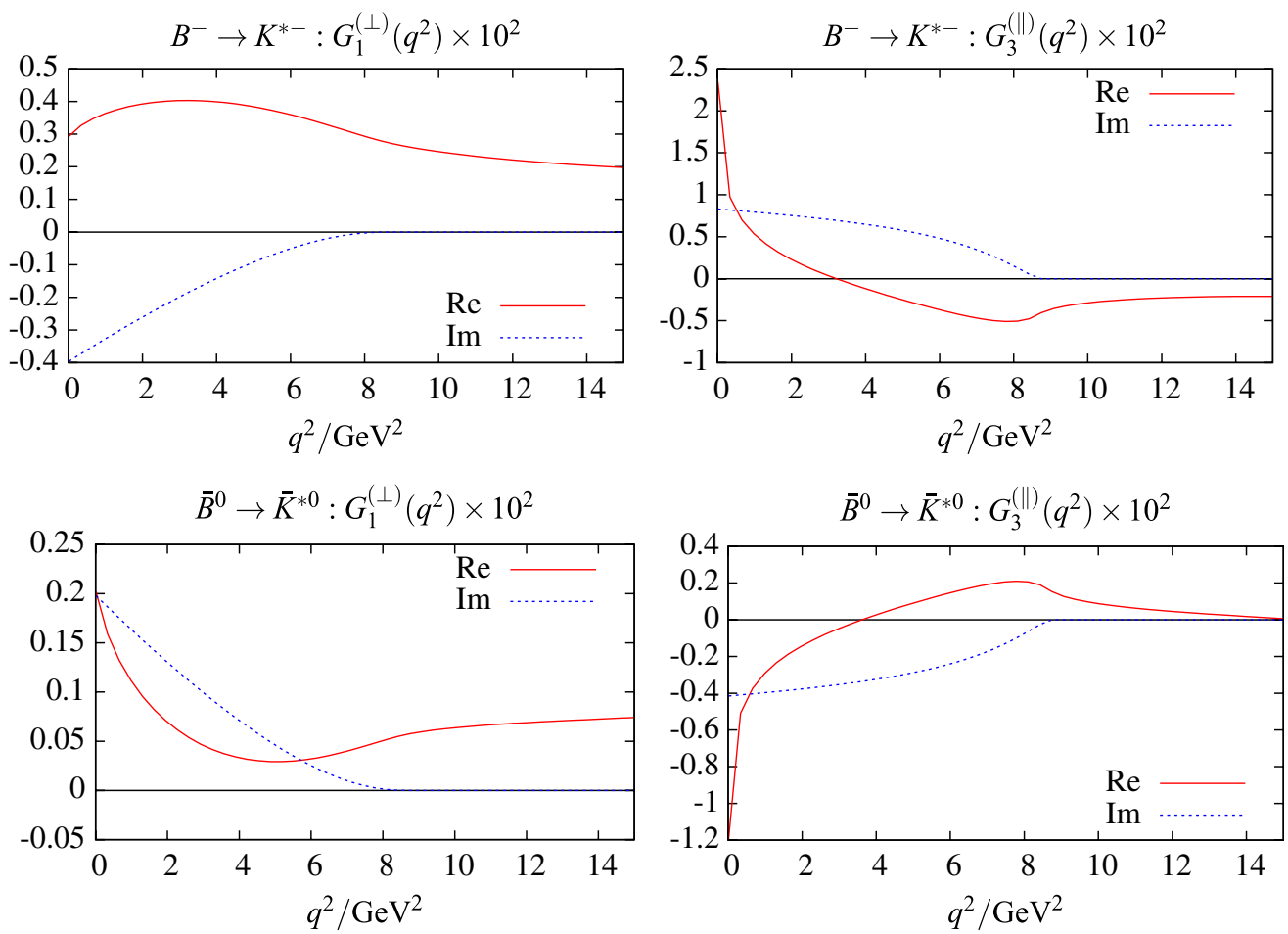

FIG. 5 (color online). Plots of $G_{1}^{(\perp)}\left(q^{2}\right)$ and $G_{3}^{(\|)}\left(q^{2}\right)$ for charged and uncharged $B$ mesons. Any other $G_{\iota}$ function where a $U$ - or $D$-type flavor is exchanged is qualitatively similar. As usual $U$ and $D$ type stand for the $u, c, t$ and $d, s, b$ flavors. For further qualitative discussion the reader is referred to Sec. IVA.

In Table III we reproduce values for $G_{1}(0)$ for the spectator contributions $G_{1}^{(s)}(0)$, the nonspectator contributions $G_{1}^{(\mathrm{ns})}(0)$, and their sum $G_{1}(0)=G_{1}^{(s)}(0)+G_{1}^{(\mathrm{ns})}(0)$, as well as ratios between the latter and the SD penguin form factors $T_{1}(0)$. Let us briefly discuss the heavy quark scaling of the various parts. ${ }^{20}$ From $T_{1}(0) \sim m_{b}^{-3 / 2}$ (as first derived in Ref. [30]) it follows that $G_{1}^{(\mathrm{ns})}(0) \sim m_{b}^{-3 / 2}$ from the formulas given in Appendix D. For $G_{1}^{(s)}$ it is useful to split the matrix elements according to whether or not the photon is emitted from the spectator:

$$
\begin{aligned}
& G_{1}^{(s)}(0)=Q_{h} G_{1}^{h,(s)}(0)+Q_{q} G_{1}^{q,(s)}(0), \\
& h \in\{b, c\}, q \in\{u, d, s\} .
\end{aligned}
$$

The discussion of Sec. VA suggests that $G_{1}^{h,(s)}(0) \sim m_{b}^{-3 / 2}$ and $G_{1}^{q,(s)}(0) \sim m_{b}^{-5 / 2}\left(\ln m_{b}+\mathcal{O}(1)\right)$. Let us discuss the numerical ratios. The ratios of $\left|G_{1}^{(\perp)}(0) / G_{1}^{(\mathrm{ns})}\right|$ are between $20 \%$ and $59 \%$ and vary considerably according to the charge and flavor of the heavy initial meson. The ratio of $\left|G_{1}^{(s)}(0) / T_{1}(0)\right|$ is around $2 \%$ for the $B$ meson and

\footnotetext{
${ }^{20} \mathrm{~A}$ word of caution seems appropriate here. In Sec. VA it is found that, for diagrams $A_{1}$ and $A_{2}$, the leading heavy quark term, including a nonexpandable logarithm in $m_{b}$, gives roughly $50 \%$ of the contribution. Whereas this points towards large corrections, it does not imply that qualitative behavior cannot be understood from the leading scaling.
}

considerably larger for the $D^{0(-)}$ at $5 \%(13 \%)$. The ratio of the total $G_{1}(0)$ to the SD part, $\left|G_{1}(0) / T_{1}(0)\right|$, is $7 \%$ for the $B$ meson and rather sizeable for the $D^{0(-)}: 21 \%$ (34\%). An interesting aspect is the comparison of the $B$ and $D$ matrix elements themselves. To obtain a meaningful answer we have to use the decomposition (26):

$$
\begin{aligned}
& R_{h}=\frac{G_{1}^{b,(\perp)}(0)[B \rightarrow \rho \gamma]}{G_{1}^{c,(\perp)}(0)[D \rightarrow \rho \gamma]}=0.14, \\
& R_{l}=\frac{G_{1}^{q,(\perp)}(0)[B \rightarrow \rho \gamma]}{G_{1}^{q,(\perp)}(0)[D \rightarrow \rho \gamma]}=0.05+0.04 i .
\end{aligned}
$$

Using the scaling behavior above we would infer that

$$
\begin{aligned}
\left|R_{h[q]}\right| & \simeq \alpha_{s}\left(\sqrt{m_{c} \Lambda_{\mathrm{had}}}\right) / \alpha_{s}\left(\sqrt{m_{b} \Lambda_{\mathrm{had}}}\right)\left(m_{c} / m_{b}\right)^{3 / 2[5 / 2]} \\
& \simeq 0.2[0.06]
\end{aligned}
$$

which is very close to the values in Eq. (27).

\section{B. Validity of computation in the $q^{2}$ range}

Let us discuss the validity of our computation in the $q^{2}$ range in more detail than in Sec. IIIC. The computation cannot be trusted when either real QCD or perturbative QCD, as employed here, ${ }^{21}$ predicts the production of

\footnotetext{
${ }^{21}$ By which we mean the LC-OPE with perturbatively computed hard scattering kernels.
} 
TABLE III. Comparison of various parts of the four characteristic types of $G_{\iota}$ functions. See Sec. IVA for comments. For the $T_{1}(0)$ form factors we use $T_{1}^{B \rightarrow \rho}(0)=0.27$ [28] for $B \rightarrow \rho$ and $T_{1}^{D \rightarrow \rho}(0)=0.7$, e.g., Ref. [29], for $D \rightarrow \rho$ as reference values. Note $G_{1}^{(s)}(0)=G_{1}^{(\perp)}(0)$ at our level of twist approximation. The ratio of $G_{1}^{(n s)}$ to $T_{1}(0)$ can directly be inferred from the formula (29).

\begin{tabular}{lcccc}
\hline \hline Type & $B^{-} \rightarrow \rho^{-} \gamma$ & $\bar{B}^{0} \rightarrow \rho^{0} \gamma$ & $D^{+} \rightarrow \rho^{+} \gamma$ & $D^{0} \rightarrow \rho^{0} \gamma$ \\
\hline$G_{1}^{(s)}(0) \cdot 10^{-2}$ & $0.29-0.39 i$ & $0.22+0.19 i$ & $-1.9+2.5 i$ & $-7.0-5.0 i$ \\
$G_{1}^{(n s)}(0) \cdot 10^{-2}$ & $0.90+1.3 i$ & $0.90+1.3 i$ & $-8.5-12 i$ & $-8.5-12 i$ \\
$G_{1}(0) \cdot 10^{-2}$ & $1.2+0.91 i$ & $1.1+1.5 i$ & $-10-9.5 i$ & $-16-17 i$ \\
$\left|G_{1}^{(s)}(0) / G_{1}^{(n s)}(0)\right|[\%]$ & 31 & 18 & 21 & 58 \\
$\left|G_{1}^{(s)}(0) / T_{1}(0)\right|[\%]$ & 2 & 1 & 4 & 12 \\
$\left|G_{1}(0) / T_{1}(0)\right|[\%]$ & 6 & 7 & 20 & 33 \\
\hline \hline
\end{tabular}

particles, which would be hadrons, and quarks and gluons, in the respective cases. This happens in real QCD when $q^{2}$ reaches the $\rho, B_{d, s}^{*}$ and $\Upsilon(\bar{b} b)$ thresholds for $J^{P C}=1^{--}$mesons. The corresponding production thresholds for perturbative QCD are of the two-valencequark type and occur at $q^{2}:\left(2 m_{q}\right)^{2},\left(m_{b}+m_{d, s}\right)^{2}$ and $\left(2 m_{b}^{2}\right)$ respectively.

As previously stated the $\rho$ threshold leads to the exclusion of the region $0<q^{2}<\left(\simeq 1 \mathrm{GeV}^{2}\right)$ for $B \rightarrow$ Vll. The quark threshold at $\left(m_{b}+m_{d, s}\right)^{2}$ indicates that the LC-OPE is not valid a few $\mathrm{GeV}$ below that value. This is the case for all diagrams except $A_{1}-A_{2}$ which do not have these thresholds and therefore the validity ought to extend a few $\mathrm{GeV}$ below $B^{*}$ resonance and thus basically to the end point of the physical region.

\section{Summary for $B(D) \rightarrow V \boldsymbol{\gamma}$}

For the reader's convenience, we briefly summarize the essential points for $B(D) \rightarrow V \gamma$ decay.

$B(D) \rightarrow V \gamma: G_{1}(0)=G_{2}(0)=G_{1}^{(\perp)}(0)+G_{1}^{(\mathrm{ns})}(0)$

with

$$
G_{1}^{(\mathrm{ns})}(0) \stackrel{(\mathrm{D} 1)}{=}\left(\frac{3 \alpha_{s}\left(m_{h}\right)}{4 \pi}\right) Q_{h} F_{8}^{(7)} T_{1}(0)
$$

where $h=b(c), Q_{b(c)}=-1 / 3(2 / 3)$ and $F_{8}^{(7)}$ are taken from Ref. [16]. The generic amplitude assumes the following form ${ }^{22}$ :

$$
\mathcal{A}(B(D) \rightarrow V \gamma) \sim\left(\mathcal{A}_{1}\left(P_{1} \cdot \epsilon\right)+\mathcal{A}_{2}\left(P_{2} \cdot \epsilon\right)\right),
$$

where $\mathcal{A}_{L, R}=\mathcal{A}_{1} \pm \mathcal{A}_{2}$ correspond to left- and righthanded photon polarizations. Our result and the leading SD penguin read

$$
\mathcal{A}_{1}=\mathcal{A}_{2}=C_{7} T_{1}(0)+C_{8} G_{1}(0)+\cdots .
$$

\footnotetext{
${ }^{22}$ The amplitudes $\mathcal{A}_{1,2}$ up to normalization are often denoted by $A_{\mathrm{PC}, \mathrm{PV}}$ in the literature.
}

Using the notation $\mathcal{O}_{7,8}^{\prime}=\left.\mathcal{O}_{7,8}\right|_{\gamma_{5} \rightarrow-\gamma_{5}}$ for the penguin operators with opposite chirality and the corresponding Wilson coefficients one gets

$$
\mathcal{A}_{1,2}=C_{7} T_{1}(0)+C_{8} G_{1}(0) \pm\left(C_{7}^{\prime} T_{1}(0)+C_{8}^{\prime} G_{1}(0)\right)+\cdots,
$$

where we have used $T_{1}(0)=T_{2}(0)$ and $G_{1}(0)=G_{2}(0)$. The former is an equality and the latter is a result of our leading twist- 2 computation.

\section{COMPARISON WITH QCD FACTORIZATION}

In this section we shall compare our results with QCDF [4]. More precisely the diagrams $A_{1}$ and $A_{2},{ }^{23}$ in Fig. 2, at $q^{2}=0$ corresponding to $Q_{q} G_{1}^{q,(s)}(0)$ (23) shall be considered where the formulas take on a rather simple form. Let us first define the quantities in question and then point towards the points we would like to investigate. We parametrize the $G_{1}$ function at $q^{2}=0$ as follows:

$$
G_{1}(0)=\underbrace{\left[\frac{\alpha_{s}}{4 \pi} \frac{C_{F}}{N_{c}} 12 \pi^{2} \frac{f_{\perp} f_{B}}{m_{B}^{2}}\right]}_{\sim m_{b}^{-5 / 2}}\left(Q_{q} X_{\perp}+Q_{b} \bar{X}_{\perp}\right),
$$

with $X_{\perp}$ as in Ref. [4],

$$
\begin{gathered}
X_{\perp}=\int_{0}^{1} \phi_{\perp}(u) x_{\perp}(u), \\
x_{\perp}^{\mathrm{QCDF}}(u)=\frac{1+\bar{u}}{3 \bar{u}^{2}}
\end{gathered}
$$

and likewise for the quantity $\bar{X}_{\perp}$. The LCSR result in this limit reads

$$
x_{\perp}^{\mathrm{LCSR}}(u)=\int_{m_{b}^{2}}^{s_{0}} d s e^{\frac{m_{B}^{2}-s}{M^{2}}} \rho(s, u)
$$

with

\footnotetext{
${ }^{23}$ Note that the sum of these two diagrams is well defined as they constitute the contribution proportional to the spectator charge.
} 


$$
\begin{aligned}
& \rho(s, u)=\underbrace{\frac{m_{b}^{2} N_{c}}{12 \pi^{2} f_{B}^{2}}}_{\equiv c m_{b}^{3}}\left[\frac{\log \left(\frac{\bar{u} s\left(m_{b}^{2}+P^{2}-s\right)}{P^{2}\left(m_{b}^{2}-u s\right)}\right)}{P^{2}-\bar{u} s}-\frac{s-m_{b}^{2}}{\bar{u} s P^{2}}\right], \\
& \bar{\rho}(s, u)=\frac{m_{b}^{2} N_{c}}{12 \pi^{2} f_{B}^{2}}\left[-\left(\frac{s-m_{b}^{2}}{u s P^{2}}-\theta\left(u s-m_{b}^{2}\right)\left(\frac{u s-m_{b}^{2}}{2 u^{2} s P^{2}}+\frac{\log \left(\frac{u s}{m_{b}^{2}}\right)}{2 u P^{2}}\right)\right)\right] .
\end{aligned}
$$

We would like to emphasize that we have computed the result in Eq. (35) anew and that we have found agreement with Ref. [4]. We have kept the contributions of diagrams $A_{3,4}$, which correspond to $\bar{X}_{\perp}$, in the expression above since their large $m_{b}$ behavior is interesting per se. A few remarks about the $m_{b}$ behavior are in order. The term in the bracket in Eq. (33) scales as $m_{b}^{-5 / 2}$, taking into account $f_{B} \sim m_{b}^{-1 / 2}$. The coefficient $c$ in Eq. (37) is $\mathcal{O}\left(m_{b}^{0}\right)$. The expression $X_{\perp}^{\mathrm{QCDF}}$ is $\mathcal{O}(1)$. The questions we would like to investigate are

(a) The presence and absence of an end-point divergence at leading order $\alpha_{s}$, for $\bar{u} \rightarrow 0$, in $X_{\perp}^{\mathrm{QCDF}}$ and $X_{\perp}^{\mathrm{LCSR}}$ respectively.

(b) In what respect $X_{\perp}^{\mathrm{QCDF}}$ and $X_{\perp}^{\mathrm{LCSR}}$ can be compared to each other.

(c) The absence and presence of an imaginary part, at leading order in $\alpha_{s}$, in $X_{\perp}^{\mathrm{QCDF}}$ and $X_{\perp}^{\mathrm{LCSR}}$ respectively.

The answers to these questions are, certainly, tied to each other. We shall begin by discussing question (a). Assuming the usual end-point behavior, ${ }^{24}$

$$
\phi_{\perp}(u) \stackrel{u \bumpeq 1}{\rightarrow} 6 \bar{u} u,
$$

the most singular part in (35),

$x_{\perp}^{\mathrm{QCDF}}=\frac{1}{3 \bar{u}^{2}}+\mathcal{O}\left(\bar{u}^{-1}\right) \Rightarrow X_{\perp}^{\mathrm{QCDF}}=2 \int_{0}^{1} \frac{d u}{\bar{u}}+$ finite

convoluted as in (34) with (38) leads to logarithmic endpoint divergence. ${ }^{25}$ The end-point configuration $u \simeq 1$ corresponds to the situation where the nonspectator quark carries all the momentum. On a purely technical level the divergent integral arises from the fact that two propagators assume the same form $1 /\left(\bar{u} m_{B}^{2}\right)$ [cf. Fig. 6 (left)], as the momentum fraction of the spectator quark is neglected due to $\Lambda_{\mathrm{QCD}} / m_{b}$ suppression. In view of this and potential transverse corrections it was advertised in Ref. [31] that for $B \rightarrow \pi \pi$ and similar cases the replacement $1 /\left(\bar{u} m_{B}^{2}\right) \rightarrow$ $1 /\left((\bar{u}+\epsilon) m_{B}^{2}\right)$ should be made $\left(\epsilon=\Lambda_{h} / m_{b}\right.$ with $\Lambda_{h}$ some hadronic scale of the order of the QCD scale) and a

\footnotetext{
${ }^{24}$ This is true to any finite order in the Gegenbauer expansion. Since the Gegenbauer polynomials are a complete set on the $[0,1]$ interval this could be changed by an infinite sum of them. This is not the currently accepted scenario.

${ }^{25}$ We note that these divergences are also regulated by $q^{2} \neq 0$ as they originate from $(\bar{u} p+q)^{2}=u q^{2}-\bar{u} u p^{2}+\bar{u}(p+q)^{2} \rightarrow$ $\bar{u}\left(m_{B}^{2}-u m_{V}^{2}\right)+u q^{2}$ but not by a finite meson final state mass.
}

correction term included to account for missing soft contributions with possible strong phases. The end-point divergent integral in (39) becomes

$$
\begin{gathered}
x_{\perp}^{\mathrm{QCDF}} \rightarrow\left(1+\rho e^{i \phi}\right) \Theta\left(\bar{u}-\frac{\Lambda_{h}}{m_{b}}\right) \frac{1}{3 \bar{u}^{2}}+\mathcal{O}\left(\bar{u}^{-1}\right) \\
\Rightarrow X_{\perp}^{\mathrm{QCDF}}=2\left(1+\rho e^{i \phi}\right) \ln \left(\frac{m_{b}}{\Lambda_{h}}\right)+\Lambda_{h} \text {-independent }
\end{gathered}
$$

with $\rho \in[0,1]$ and $\phi \in[0,2 \pi]$ being numbers parametrizing the above mentioned corrections. Thus changes can be expected if the heavy quark limit is not assumed as is the case in LCSR. Yet the question we would like to address is whether there are qualitative differences beyond the behavior of the rhs in Eqs. (40) and (41).

In the LCSR computation there is only one propagator with manifest $1 /\left(\bar{u} m_{B}^{2}\right)$ behavior; cf. Fig. 6 . Thus we ask the question: is there another one hidden in the loop? The answer to that is no as it would correspond to a power IR divergence, whereas it is known that in four dimensions IR singularities, be they soft or collinear, are at worst logarithmic in nature, e.g., Ref. [32]. The smoother behavior of the diagram in Fig. 6 (left) with respect to the QCDF result Fig. 6 (right) is in line with the improved IR behavior of inclusive processes as manifested in the classic IR-cancellation theorems of the Bloch-Nordsieck and Kinoshita-Lee-Nauenberg type [32]. ${ }^{26}$ Inspection of the diagrams in Fig. 6 reveals that there can at most be a collinear divergence in the limit $\bar{u} \rightarrow 0$ and $p^{2}=q^{2}=0$. The potential end-point sensitive terms are parametrized as follows:

$$
x_{\perp}^{\mathrm{LCSR}} \sim \alpha_{\perp} \frac{\ln (\bar{u})}{\bar{u}}+\beta_{\perp} \ln (\bar{u})+\gamma_{\perp} \frac{1}{\bar{u}} .
$$

Note that they are all integrable assuming the DA Eq. (38). From Eq. (37) $)^{27}$ it is found that $\alpha_{\perp}=0, \beta_{\perp} \neq 0, \gamma_{\perp} \neq 0$. The absence of the most singular term $\ln (\bar{u}) / \bar{u}$ appears to be accidental; such terms are present in the $P / V^{\|}$contribution.

\footnotetext{
${ }^{26}$ At this point it is more inclusive because we sum over all states with $B$-meson quantum numbers and because there are additional LD contributions [Fig. 4 (left)]. The former will be removed once the correlation function is inserted into modified dispersion integral (17). It remains to be investigated what happens when the $m_{b}$ scaling of $s_{0}, m_{B}$ and $M$ is made explicit as done in Sec. VA.

${ }^{27}$ Integration over $d s$ is not going to change anything at this point.
} 

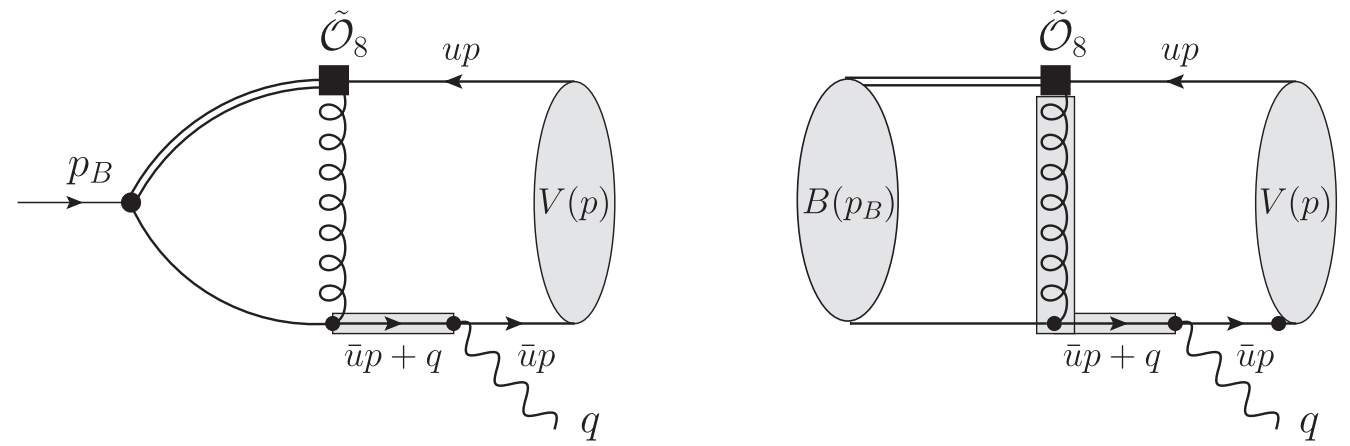

FIG. 6. The shaded propagators that scale like $1 /\left(\bar{u} m_{B}^{2}\right)$ in both figures. (left) Diagram of LCSR or the LC-OPE respectively. (right) Diagram in QCDF. Thus $x_{\perp}^{\mathrm{QCDF}} \sim 1 / \bar{u}^{2}$ and $x_{\perp}^{\mathrm{LCSR}} \sim \ln (\bar{u}) / \bar{u}$ at worst, as explained in the text.

In summary, the end-point behavior of the $x_{\perp}^{\mathrm{LCSR}}$ (42) differs from $x_{\perp}^{\mathrm{QCDF}}(39)$ even when finite $m_{b}$ effects are added by hand (40).

Before attempting an interpretation of this difference we should try to reflect on question (b), namely to what degree it makes sense to compare the QCDF and the LCSR result at face value.

We advocate that, within the approximations, the QCDF contribution is contained in the LCSR result but the converse is not true. For example the gluon in Fig. 6 (right) is not necessarily the hard gluon of QCDF but can also be a gluon that hadronizes into a 3-particle $(q s)_{0^{ \pm}}$state as shown in Fig. 4 (left). Moreover there are cuts of the 3-particle type for the $B$ meson as well; cf. Fig. 3. Possibly it is helpful, at this point, to note that there is a crucial difference between the two approaches. In QCDF one computes a specific subprocess and the corresponding scaling of the momenta leads to a clear physical picture of the dynamics of that subprocess, whereas in LCSR one computes a correlation function, in a domain where it is believed to be valid, and extracts the matrix element by suitable methods such as dispersion relation and Borel transformation. Thus the physical parton configurations are, generically, not immediately deducible from the correlation function.

In summary the LCSR result is not end-point divergent, yet it is sensitive to the end point. ${ }^{28}$ We have seen that the amendment (40) is not enough to obtain a similar qualitative behavior of $x_{\perp}^{\mathrm{QCDF}}$ and $x_{\perp}^{\mathrm{LCSR}}$. Whether or not this is due to the fact that $x_{\perp}^{\mathrm{LCSR}}$ constitutes in addition to the physics present in $x_{\perp}^{\mathrm{QCDF}}$ a LD part [Fig. 4 (left)] is a question that we did not address. The question of why the QCDF contribution does not admit, in its current form, a heavy quark expansion can be illuminated by investigating what

\footnotetext{
${ }^{28}$ At leading order in $\alpha_{s}$ the most sensitive term is $\Delta=$ $\int 6 u \bar{u} \frac{1}{\bar{u}}=3 \sum_{n \geq 0}(-1)^{n} a_{n}$ where $a_{n}$ are the Gegenbauer moments, e.g., Ref. [1]. Explicit computations of the Gegenbauer moments as well as an investigation of the pion form factor [33] show that the influence of the Gegenbauer moments on this quantity is rather moderate (at the 10\%-20\% level).
}

happens when a LCSR heavy quark expansion is attempted. This is the goal of the next subsection.

\section{A. Heavy quark limit and the dependence on the value of $m_{b}$}

In this section we would like to investigate whether the two approaches behave similarly in the heavy quark limit. Although this cannot be done in an absolutely transparent way, a rescaling in the heavy quark mass ${ }^{29} m_{b}$ has been proposed in [30,34]:

$$
\begin{gathered}
m_{B} \rightarrow m_{b}+\bar{\Lambda}, \quad s_{0} \rightarrow m_{b}^{2}+2 m_{b} \omega_{0}, \\
M^{2} \rightarrow 2 m_{b} \tau,
\end{gathered}
$$

where $\bar{\Lambda}, \omega_{0}$ and $\tau$ are all hadronic scales of which $\bar{\Lambda}$ is, of course, rather well known. In many cases this has reproduced the leading order behavior from a proper heavy quark treatment of the same quantity. The expansions in $m_{B}$ and $s_{0}$ are of leading order and the Borel mass $M^{2}$ is adjusted such that the exponential is free of powers of $m_{b}$. The expression $x^{\mathrm{LCSR}}$ can then be rewritten in terms of the dimensionless integration variable $z$ :

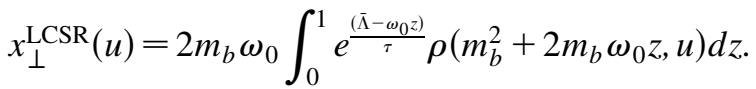

Using the asymptotic DA $\phi_{\perp}(u)=6 u \bar{u}$ in (34), integrating over $d u$ and isolating a nonexpandable logarithm we get

$$
\begin{aligned}
X_{\perp}^{\mathrm{LCSR}}= & \underbrace{\left[\frac{N_{c} \omega_{0}^{2}}{f_{B}^{2} \pi^{2}}\right]\left\{\frac{2 \omega_{0}}{m_{b}}\left(\left(\ln \left(\frac{m_{b}}{2 \omega_{0}}\right)-i \pi\right)\left\langle z^{2}\right\rangle-\left\langle z^{2} \ln z\right\rangle\right)\right.}_{\left(X_{\perp}^{\mathrm{LCSR}}\right)^{(0)}} \\
& \left.+\mathcal{O}\left(\frac{\Lambda_{\mathrm{QCD}}^{2}}{m_{b}^{2}}\right)\right\} \\
\bar{X}_{\perp}^{\mathrm{LCSR}}= & {\left[\frac{N_{c} \omega_{0}^{2}}{f_{B}^{2} \pi^{2}}\right]\left\{\left(\langle z\rangle\left(\frac{2 \bar{\Lambda}}{m_{b}}-1\right)+\frac{2 \omega_{0}}{m_{b}}\left\langle z^{2}\right\rangle\right)+\mathcal{O}\left(\frac{\Lambda_{\mathrm{QCD}}^{2}}{m_{b}^{2}}\right)\right\}, }
\end{aligned}
$$

\footnotetext{
${ }^{29}$ We refrain from rescaling $f_{B} \rightarrow\left(f_{B}\right)_{\text {stat }} m_{b}^{-1 / 2}$. We shall simply use this known scaling behavior in what follows.
} 

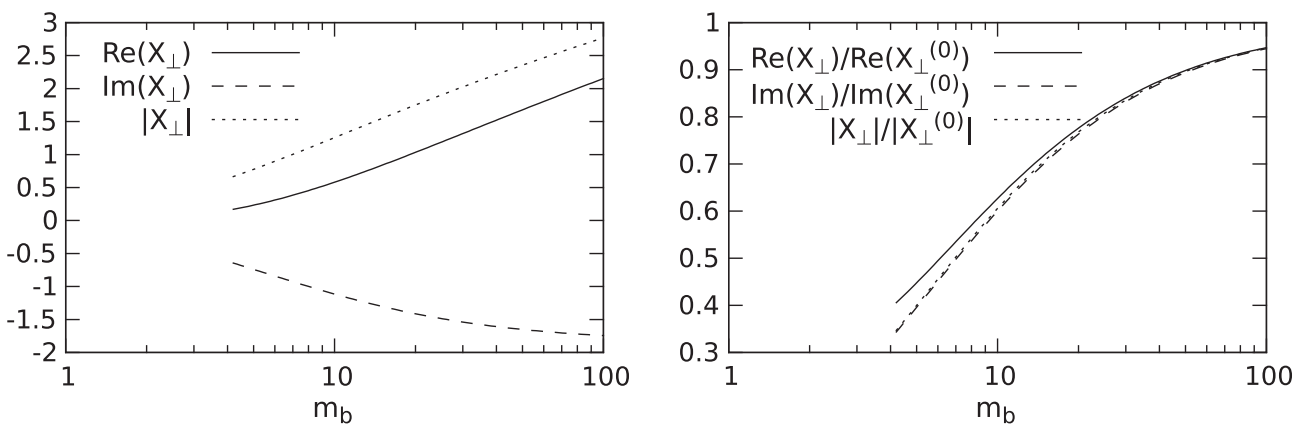

FIG. 7. (left) Absolute value, real and imaginary part of $X_{\perp}^{\mathrm{LSCR}}$ as a function of $m_{b}$ assuming the rescaling (43). The plot makes it apparent that the hierarchy of the real and imaginary parts is rather dependent on the actual value of $m_{b}$. (right) Ratio of the asymptotic expression $\left(X_{\perp}^{\mathrm{LCSR}}\right)^{(0)}$ in (45) over the expression including all $m_{b}$ corrections within the rescaling (43). Note that nonleading order corrections decrease the quantity $X_{\perp}$.

with $\langle f(z)\rangle=\int_{0}^{1} e^{\frac{\left(\bar{\Lambda}-\omega_{0} z\right)}{\tau}} f(z) d z$ being a number of order 1 . A few remarks are in order:

(i) From the appearance of the imaginary part at leading order it would seem in the heavy quark limit (43) that the QCDF and LCSR computations cannot be compared, as the former are real. This would suggest that the LD contributions [cf. Fig. 4 (left)] responsible for the $C P$-even phases do not seem to be suppressed in the heavy quark limit for spectator emission.

(ii) Equation (45) suggests, using the notation as in Eq. (26), that

$$
\begin{aligned}
& G_{1}^{b}(0) \sim m_{b}^{-3 / 2} \\
& G_{1}^{q}(0) \sim m_{b}^{-5 / 2}\left(\ln m_{b}+\mathcal{O}(1)\right) .
\end{aligned}
$$

These scaling behaviors are in line with Refs. [2,3] for $G_{1}^{b}(0)$ and Ref. [4] for $G_{1}^{q}(0)$. The end-point divergence can be associated with the nonexpandable logarithm to be discussed below.

(iii) The $\ln m_{b}$ term signals that the result, using the rescaling (43), is not expandable in powers of $1 / m_{b}$. This statement is of course dependent on the behavior of the DA at the end point $u \simeq 1$ (38). This can be further illustrated by first expanding the density $\rho$ in Eq. (44) in inverse powers of the heavy quark mass. To leading order we get

$$
\begin{aligned}
& \operatorname{Re}[\rho]=\frac{2 c \omega_{0}^{2} z^{2}}{m_{b}} \frac{1+\bar{u}}{\bar{u}^{2}}, \\
& \operatorname{Im}[\rho]=-\frac{c m_{b} \pi}{u} \theta\left(u-\left(1-\frac{2 \omega_{0} z}{m_{b}}\right)\right) \\
& \operatorname{Re}[\bar{\rho}]=-\frac{2 c \omega_{0} z}{u}\left(1-\frac{2 \bar{\Lambda}}{m_{b}}-\frac{2 \omega_{0}}{m_{b}} z\right), \\
& \operatorname{Im}[\bar{\rho}]=0,
\end{aligned}
$$

up to order $\mathcal{O}\left(\Lambda_{\mathrm{QCD}}^{2} / m_{b}^{2}\right)$. Thus one recovers the end-point singularity of the QCDF result. Note that the difference in powers of $m_{b}$ and $z$ in the real and imaginary parts is only apparent or compensated by the narrowness of the resulting $d u$ integration interval. Further expansion in powers of $m_{b}$ in the real part leads to more and more end-point divergent expression: $\operatorname{Re}[\rho] \sim \frac{1}{m_{b}^{n}} \frac{1}{\bar{u}^{n+1}}$. This originates from the term $u s-m_{b}^{2}$ in the logarithm in Eq. (37).

(iv) The rescaling (43) allows us to investigate the numerical dependence of the real and imaginary parts on the mass $m_{b}$. As can be inferred from Fig. 7 (left) the smallness of the real part with respect to the imaginary part at $m_{b} \simeq 4.6 \mathrm{GeV}$ is rather accidental.

(v) Information on the convergence of the $1 / m_{b}$ expansion can be inferred from Fig. 7 (right), though the cautionary remarks above and below Eq. (43) should be kept in mind.

\section{SUMMARY AND CONCLUSIONS}

In this work we have reported on the computation of $\mathcal{O}_{8}$ matrix elements between heavy pseudoscalar $B$ and $D$ states and a light vector and pseudoscalar state and an off-shell photon by using the method of LCSR at leading twist-2 and leading $\alpha_{s}$. We have defined scalar functions of the photon momentum invariant $G_{1,2,3}\left(q^{2}\right)$ and $G_{T}\left(q^{2}\right)$ Eqs. (3) and (4) such that they parallel the well-known penguin tensor form factors $T_{1,2,3}\left(q^{2}\right)$ and $f_{T}\left(q^{2}\right)$; cf. Eq. (8). Central values for all flavor transitions, with the exception of $\eta$ and $\eta^{\prime}$, are presented in Table II; plots of the four characteristic cases in Fig. 5 are presented in Sec. IV. A remarkable feature is the large $C P$-even (strong) phase for which we give a LD interpretation in Sec. IIIC (cf. Fig. 4). This fact, as well as the plots, makes it clear why we refer to $G_{\iota}\left(q^{2}\right)$ as matrix elements rather than form factors. Comparison of various contributions such as spectator, nonspectator, and SD penguin photon emission can be found in Table III. Let us note that the $G_{\iota}\left(q^{2}\right)$ functions are relevant for asymmetries of isospin- [26] and CP-type (depending on new weak phases) [9] rather than branching ratios. 
In Sec. V we compare our computation with QCDF. The comparison is not straightforward as the LCSRs contrary to QCDF are not tailored around a heavy quark expansion and second LCSRs contain LD contributions of the type shown in Fig. 4 (left) which are not present in leading order QCDF. The LCSR computation does not suffer from end-point divergences which we trace back to the fact that IR divergences are at worst logarithmic in four dimensions. When a heavy quark extrapolation of the LCSR result is attempted (cf. Sec. VA), a logarithm of $m_{b}$ appears which might be taken as an indication towards potential difficulties of the $m_{b}$ expansion, e.g., end-point divergences. ${ }^{30}$ Whether or not an approach can be devised to deal with these end-point divergences in the heavy quark limit is an interesting problem per se. Recent approaches known under the names of collinear anomaly [35] and rapidity renormalization group [36] might give rise to further developments, leading to a consistent treatment of end-point divergences in the heavy quark limit.

A remarkable feature on the technical side of our computation is the appearance of a complex anomalous threshold on the physical Riemann sheet for which we give various viewpoints and derivations in Appendix $\mathrm{H} 1$. The anomalous threshold is associated, in the three point function, with all three propagators being on the mass shell and therefore is not related to the intermediate $B$-meson state. The crucial point, for the physics, is that the anomalous thresholds are well isolated from the $m_{B}$ pole. This results in an exponential as well as oscillatory suppression by the Borel parameter such that the extraction of the matrix element is not affected considerably.

We shall add a paragraph contemplating on the size of the isospin asymmetry in $b \rightarrow q \gamma$ due to $\mathcal{O}_{8}$, interfering with the leading $\mathcal{O}_{7}$-part, in the inclusive and exclusive case. In the former this was investigated in Ref. [37] by means of a vaccum saturation approximation and it is found that,

$$
\begin{aligned}
\left.a_{I}^{\overline{0}-}\left(X_{s} \gamma\right)\right|_{O_{8}} & =\frac{\Gamma\left(\bar{B}^{0} \rightarrow X_{s} \gamma\right)-\Gamma\left(B^{-} \rightarrow X_{s} \gamma\right)}{\Gamma\left(\bar{B}^{0} \rightarrow X_{s} \gamma\right)+\Gamma\left(B^{-} \rightarrow X_{s} \gamma\right)} \\
& =-0.05\left(\frac{0.5 \mathrm{Gev}}{\lambda_{B}}\right)^{2}
\end{aligned}
$$

The symbol $\lambda_{B}$ corresponds to the first inverse moment of the $B$-meson DA whose uncertainty leads to the authors of Ref. [37] to attribute a spread of $-0.02--0.19$ to (47). For the exclusive case we find, using our work,

\footnotetext{
${ }^{30}$ When in the same limit the density of the collinear momentum fraction is expanded in $1 / m_{b}$ then indeed the same behavior as in QCDF is found. It is worthwhile that qualitative differences between the two approaches remain, even in that case, for the reason mentioned above.
}

$$
\begin{aligned}
\left.a_{1}^{\overline{0}-}\left(K^{*} \gamma\right)\right|_{O_{8}} & =\frac{C_{8} \operatorname{Re}\left[Q_{d} G_{1}^{\bar{B}^{0} \rightarrow \bar{K}^{* 0} \gamma}(0)-Q_{u} G_{1}^{B^{-} \rightarrow K^{*-} \gamma}(0)\right]}{C_{7} T_{1}^{B \rightarrow K^{*} \gamma}(0)} \\
& =-0.004(2) .
\end{aligned}
$$

We have used $G_{1}(0)$ from Table II, $T_{1}(0) \simeq 0.33$ [28], $C_{7} \simeq$ -0.36 , and $C_{8} \simeq-0.16$ [16]. It is noted that the sign of the effect is the same but the estimate of the inclusive case is somewhat higher even given the uncertainty. Since experimentally the inclusive rate is a sum of exclusive rates, the numbers in Eqs. (47) and (48) indicate that higher states than the $K^{*}$ in the spectrum are more prone to isospin violation originating from $\mathcal{O}_{8}$. At last it might be of interest to quote the current experimental averages [38] $a_{I}^{\overline{0}-}\left(X_{s} \gamma\right)=-0.01(6)$ and $a_{I}^{\overline{0}-}\left(K^{*} \gamma\right)=0.052(26)$. The isospin asymmetry in $B \rightarrow K^{*} \gamma$ is dominated by weak annihilation (c.a. 5\%) in the Standard Model [4] and from Eq. (48) we infer that the $\mathcal{O}_{8}$ contribution is rather small. For the inclusive case matters are different as weak annihilation, by which we mean contributions from four-Fermi operators, is suppressed by powers of $m_{b}$ in the operator product expansion such that $\mathcal{O}_{8}$ might be the leading effect. The latter picture is consistent with the theoretical and experimental findings quoted above.

\section{ACKNOWLEDGMENTS}

We are grateful to Guido Bell, Vladimir Braun, Thorsten Feldmann, Sebastian Jäger, Andreas Jüttner, Mikolai Misiak, Matthias Neubert, Douglas Ross, Christopher Sachrajda, Christopher Smith and Stefan Weinzierl for discussions on various aspects at various stages of the project. R.Z. gratefully acknowledges the support of an advanced STFC fellowship.

\section{APPENDIX A: LC-OPE RESULTS OF THE CORRELATION FUNCTION $\Pi^{V, P}$}

Below we present the results of the LC-OPE for the correlation functions for the vector and pseudoscalar cases using the decompositions in Eq. (20). We shall use the same decomposition as in Eq. (23),

$$
g_{i}^{(s)}=g_{i}^{(\perp)}+g_{i}^{(\|)}+\cdots, \quad i=0 \ldots 3, \quad g_{T}^{(s)}=g_{T}^{(P)}
$$

for the various contributions on the DA (F1) parts. The dots stand for higher twist contributions such as the photon DA discussed in the next Appendix. In order to present our results in a compact way we introduce the following abbreviations for the PV functions:

$$
\begin{aligned}
& B_{a}=B_{0}\left(u\left(p_{B}^{2}-P^{2}\right), 0, m_{b}^{2}\right), \\
& B_{b}=B_{0}\left(p_{B}^{2}-P^{2}, 0, m_{b}^{2}\right), \\
& B_{c}=B_{0}\left(u p_{B}^{2}+\bar{u} q^{2}, 0, m_{b}^{2}\right), \\
& B_{d}=B_{0}\left(p_{B}^{2}, 0, m_{b}^{2}\right),
\end{aligned}
$$


$C_{a}=C_{0}\left(p_{B}^{2}, u\left(p_{B}^{2}-P^{2}\right), \bar{u} P^{2}+u q^{2}, 0, m_{b}^{2}, 0\right)$,

$C_{b}=C_{0}\left(p_{B}^{2}, p_{B}^{2}-P^{2}, q^{2}, 0, m_{b}^{2}, 0\right)$,

$C_{c}=C_{0}\left(u p_{B}^{2}+\bar{u} q^{2}, u\left(p_{B}^{2}-P^{2}\right), q^{2}, m_{b}^{2}, 0, m_{b}^{2}\right)$,

$C_{d}=C_{0}\left(p_{B}^{2}, p_{B}^{2}-P^{2}, q^{2}, m_{b}^{2}, 0, m_{b}^{2}\right)$.

Note we have only listed the PV functions which depend on $p_{B}^{2}$ as the other ones do not enter the dispersion representation. Moreover the functions on the right correspond to the functions on the left at $u=1$.

\section{1. $V_{\perp}$ transverse}

We find that for the transverse parts the Lorentz projections satisfy

$$
\begin{gathered}
g_{2}^{(\perp)}=\left(1-q^{2} / P^{2}\right) g_{3}^{(\perp)}, \quad g_{2}^{(\perp)}=\left(1-q^{2} / P^{2}\right) g_{1}^{(\perp)}, \\
g_{0}^{(\perp)}=0 .
\end{gathered}
$$

The second relation is a LEET $[3,27]$ relation. It can be explained in a straightforward way at the level of the $\phi_{\perp}$-distribution in use. We may factor out the perpendicular $K^{*}$ DA from the amplitude $\mathcal{A}^{* \mu}(V)$ to give,

$$
\mathcal{A} * \mu(V)=\operatorname{Tr}\left\{\dot{m} \not I^{\mu}\right\}+\cdots,
$$

since the projector is proportional to $\not p \eta^{*}(\mathrm{~F} 1)$. The dots stand for contributions from other terms in the $K^{*}$ lightcone expansion. $I^{\mu}$ may generally be written as

$I^{\mu}(V)=\left[I_{0}^{\mu}+I_{1} \not \partial \gamma^{\mu}+I_{2} \not \gamma^{\mu}+I_{3} \not \not q\right]\left(1-\gamma_{5}\right)$,

where terms with an odd number of matrices have been excluded because they do not contribute to (A3). Inserting this form into (A3) then gives

$$
\operatorname{Tr}\left\{\not \not \not I^{\mu}\right\}=I_{2} \operatorname{Tr}\left\{\not \not \not \not \phi \gamma^{\mu}\left(1-\gamma_{5}\right)\right\},
$$

and hence there is only a single scalar amplitude which contributes to the result. Evaluating the trace in our basis (E1) yields the identity

$$
G_{2}^{(\perp)}\left(q^{2}\right)+\frac{q^{2}}{m_{B}^{2}} G_{3}^{(\perp)}\left(q^{2}\right)=G_{1}^{(\perp)}\left(q^{2}\right) .
$$

As previously noted $G_{2}^{(\perp)}\left(q^{2}\right)=\left(1-q^{2} / m_{B}^{2}\right) G_{3}^{(\perp)}\left(q^{2}\right)$ so it follows that $G_{1}^{(\perp)}\left(q^{2}\right)=G_{3}^{(\perp)}\left(q^{2}\right)$ which shows consistency between the two equations in Eq. (A2). The first relation is of a more general type which we would like to explain below: Decomposing the following matrix element,

$$
\begin{aligned}
& \left\langle\gamma^{*}(q, \rho) V(p, \eta)\left|H_{\mathrm{eff}}\right| \bar{B}\left(p_{B}\right)\right\rangle \\
& \quad=X_{1}\left(q^{2}\right) P_{1}^{\rho}+X_{2}\left(q^{2}\right) P_{2}^{\rho}+X_{3}\left(q^{2}\right) P_{3}^{\rho},
\end{aligned}
$$

the relation

$$
X_{2}-\left(1-\frac{q^{2}}{m_{B}^{2}}\right) X_{3}=\mathcal{O}\left(m_{V}\right),
$$

must be true in order to cancel an explicit factor $1 / m_{V}$ in the decay rate [26]. More precisely, by this argument we preclude power divergences which cannot be there as IR divergences are at worst logarithmic in four dimensions, as mentioned previously. Thus, for any projection which does not contain an explicit $m_{V}$ factor in its definition, e.g., $\phi_{\perp}$ but not $\phi_{\|}$, the relation holds up to $\mathcal{O}\left(m_{V}^{2}\right)$. e.g., $G_{2}^{(\perp)}=$ $\left(1-q^{2} / m_{B}^{2}\right) G_{3}^{(\perp)}+\mathcal{O}\left(m_{V}^{2}\right)$. Since we neglect $m_{V}^{2}$ altogether the first relation in Eq. (A2) is a necessary outcome.

We parametrize the result $g_{1}^{(\perp)}\left(q^{2}\right)$ as

$k_{V}^{-1} g_{1}^{(\perp)}\left(q^{2}\right)=\frac{\alpha_{s}}{4 \pi} C_{F}\left(-\frac{1}{2}\right) f_{V}^{\perp} m_{b}^{2} Q_{b} \int_{0}^{1} d u t_{H}^{(\perp)}(u) \phi_{\perp}(u)$,

where the $t_{H}^{(\perp)}(u)$ corresponds to the hard kernel and is given in terms of PV functions

$$
t_{H}^{(\perp)}(u)=\sum_{i=a}^{d}\left(b_{i}^{\perp} B_{i}+c_{i}^{\perp} C_{i}\right),
$$

where the sum extends alphabetically from $a$ to $d$. The only nonzero coefficients are

$$
\begin{aligned}
\left(b_{a}^{\perp}, b_{c}^{\perp}, b_{d}^{\perp}\right) & =\left(\frac{q_{R}}{u q^{2}+\bar{u} P^{2}}, \frac{1}{\bar{u} q^{2}+u P^{2}}, 2\left(b_{a}^{\perp}+b_{c}^{\perp}\right)\right), \\
\left(c_{a}^{\perp}, c_{c}^{\perp}\right) & =\left(-2 q_{R},-1\right),
\end{aligned}
$$

with $q_{R} \equiv Q_{q} / Q_{b}$ being the charge ratio.

\section{2. $V_{\|}$longitudinal}

The computation of $V_{\|}$is in principle highly nontrivial due to the extra coordinates $x$ appearing in front of the integral in Eq. (F1). We shall employ though the so-called ultrarelativistic limit,

$$
\eta(p)_{\alpha} \rightarrow \frac{1}{m_{V}}\left(p_{\alpha}+\mathcal{O}\left(\frac{m_{V}^{2}}{E_{V}^{2}}\right) \zeta_{\alpha}\right)
$$

which is correct up to the relativistic correction as indicated and the vector $\zeta$ is a linear combination of $p$ and $\eta$. In this limit, using the DA as given in Appendix $\mathrm{F}$, the $V_{\|}$ and $P$ contributions are identical up to the replacements $f_{V}^{\|} \rightarrow-i f_{P}$ and $\phi_{\|} \rightarrow \phi_{P}$, as can easily be understood by commuting the $\gamma_{5}$ through the diagram until it is "annihilated" by $\left(1+\gamma_{5}\right) \gamma_{5}=\left(1+\gamma_{5}\right)$ which originates from $\tilde{\mathcal{O}}_{8}$. Noting that in the ultrarelativistic limit

$$
P_{1} \rightarrow 0,\left.\quad P_{2} \rightarrow c P_{3}\right|_{\eta \rightarrow p / m_{V}}
$$

with $c$ a constant it is clear ${ }^{31}$ that only $g_{3}$ receives a contribution. Taking further into account Eq. (E4) one gets

\footnotetext{
${ }^{31}$ The more careful reader might want to know that $P_{2}$ corresponds to a term which is linearly dependent and one that is linearly independent of $P_{3}$. In the limit the latter vanishes.
} 
TABLE IV. $\quad q^{2}$ dependence of the $G_{1}^{(\perp)}\left(q^{2}\right)$ and $G_{3}^{(\|)}\left(q^{2}\right)$ functions for the four characteristic cases depending on whether the initial state is $B^{-}, \bar{B}^{0}, D^{0}$ or $D^{+}$type. The tables can be requested from the authors.

\begin{tabular}{|c|c|c|c|c|}
\hline & $G_{1}^{(\perp)} \times 10^{2}$ & $G_{3}^{(\mathrm{ll})} \times 10^{2}$ & $G_{1}^{(\perp)} \times 10^{2}$ & $G_{3}^{(\mathrm{II})} \times 10^{2}$ \\
\hline$Q^{2}$ & $B^{-} \rightarrow K^{*-}$ & $B^{-} \rightarrow K^{*-}$ & $\bar{B}^{0} \rightarrow \bar{K}^{* 0}$ & $\bar{B}^{0} \rightarrow \bar{K}^{* 0}$ \\
\hline 0.010 & $0.2931-0.3960 i$ & $2.3443+0.8303 i$ & $0.2022+0.1980 i$ & $-1.1952-0.4151 i$ \\
\hline 0.261 & $0.3204-0.3781 i$ & $1.0673+0.8213 i$ & $0.1661+0.1890 i$ & $-0.5574-0.4107 i$ \\
\hline 0.512 & $0.3384-0.3604 i$ & $0.7999+0.8122 i$ & $0.1431+0.1802 i$ & $-0.4243-0.4061 i$ \\
\hline 0.764 & $0.3526-0.3429 i$ & $0.6388+0.8029 i$ & $0.1251+0.1715 i$ & $-0.3443-0.4014 i$ \\
\hline 1.015 & $0.3641-0.3257 i$ & $0.5217+0.7933 i$ & $0.1102+0.1629 i$ & $-0.2863-0.3966 i$ \\
\hline 1.266 & $0.3736-0.3087 i$ & $0.4286+0.7834 i$ & $0.0975+0.1544 i$ & $-0.2403-0.3917 i$ \\
\hline 1.517 & $0.3815-0.2920 i$ & $0.3508+0.7732 i$ & $0.0866+0.1460 i$ & $-0.2020-0.3866 i$ \\
\hline 1.768 & $0.3878-0.2755 i$ & $0.2834+0.7628 i$ & $0.0771+0.1378 i$ & $-0.1688-0.3814 i$ \\
\hline 2.020 & $0.3929-0.2593 i$ & $0.2235+0.7519 i$ & $0.0689+0.1297 i$ & $-0.1395-0.3760 i$ \\
\hline 2.271 & $0.3969-0.2434 i$ & $0.1693+0.7407 i$ & $0.0617+0.1217 i$ & $-0.1129-0.3703 i$ \\
\hline 2.522 & $0.3998-0.2277 i$ & $0.1196+0.7290 i$ & $0.0554+0.1139 i$ & $-0.0887-0.3645 i$ \\
\hline 2.773 & $0.4018-0.2124 i$ & $0.0734+0.7168 i$ & $0.0499+0.1062 i$ & $-0.0662-0.3584 i$ \\
\hline 3.024 & $0.4028-0.1974 i$ & $0.0300+0.7041 i$ & $0.0453+0.0987 i$ & $-0.0451-0.3521 i$ \\
\hline 3.275 & $0.4030-0.1827 i$ & $-0.0110+0.6908 i$ & $0.0413+0.0913 i$ & $-0.0253-0.3454 i$ \\
\hline 3.527 & $0.4024-0.1683 i$ & $-0.0500+0.6768 i$ & $0.0379+0.0842 i$ & $-0.0064-0.3384 i$ \\
\hline 4.786 & $0.3883-0.1024 i$ & $-0.2248+0.5935 i$ & $0.0295+0.0512 i$ & $0.0775-0.2967 i$ \\
\hline 6.046 & $0.3586-0.0489 i$ & $-0.3754+0.4758 i$ & $0.0323+0.0245 i$ & $0.1488-0.2379 i$ \\
\hline 7.305 & $0.3177-0.0129 i$ & $-0.4908+0.2946 i$ & $0.0431+0.0065 i$ & $0.2019-0.1473 i$ \\
\hline 8.565 & $0.2758+0.0000 i$ & $-0.4519+0.0224 i$ & $0.0562-0.0000 i$ & $0.1770-0.0112 i$ \\
\hline 9.824 & $0.2492+0.0000 i$ & $-0.2972+0.0000 i$ & $0.0630-0.0000 i$ & $0.0933-0.0000 i$ \\
\hline 11.084 & $0.2312-0.0000 i$ & $-0.2485-0.0000 i$ & $0.0669+0.0000 i$ & $0.0613+0.0000 i$ \\
\hline 12.343 & $0.2176-0.0000 i$ & $-0.2243-0.0000 i$ & $0.0696+0.0000 i$ & $0.0400+0.0000 i$ \\
\hline 13.603 & $0.2070-0.0000 i$ & $-0.2128+0.0000 i$ & $0.0718+0.0000 i$ & $0.0230-0.0000 i$ \\
\hline 14.862 & $0.1986+0.0000 i$ & $-0.2101+0.0000 i$ & $0.0740-0.0000 i$ & $0.0076-0.0000 i$ \\
\hline 16.122 & $0.1921-0.0000 i$ & $-0.2147-0.0000 i$ & $0.0763+0.0000 i$ & $-0.0080+0.0000 i$ \\
\hline 17.381 & $0.1873-0.0000 i$ & $-0.2267+0.0000 i$ & $0.0790+0.0000 i$ & $-0.0252-0.0000 i$ \\
\hline 18.641 & $0.1843+0.0000 i$ & $-0.2475+0.0000 i$ & $0.0824-0.0000 i$ & $-0.0459-0.0000 i$ \\
\hline 19.900 & $0.1831-0.0000 i$ & $-0.2803+0.0000 i$ & $0.0869+0.0000 i$ & $-0.0725-0.0000 i$ \\
\hline \multirow[t]{2}{*}{21.160} & $0.1844-0.0000 i$ & $-0.3310-0.0000 i$ & $0.0932+0.0000 i$ & $-0.1097+0.0000 i$ \\
\hline & $D^{0} \rightarrow \rho^{0}$ & $D^{0} \rightarrow \rho^{0}$ & $D^{+} \rightarrow \rho^{+}$ & $D^{+} \rightarrow \rho^{+}$ \\
\hline 0.010 & $-7.0027-4.9787 i$ & $14.939+2.507 i$ & $-1.9295+2.4893 i$ & $19.589-1.254 i$ \\
\hline 0.048 & $-6.5207-4.7048 i$ & $10.506+2.673 i$ & $-1.8309+2.3524 i$ & $0.5204-1.3366 i$ \\
\hline 0.087 & $-6.2041-4.4945 i$ & $8.9314+2.7462 i$ & $-1.7662+2.2472 i$ & $-1.0918-1.3731 i$ \\
\hline 0.125 & $-5.9599-4.3583 i$ & $7.9497+2.9176 i$ & $-1.7163+2.1792 i$ & $-1.4961-1.4588 i$ \\
\hline 0.163 & $-5.7571-4.2099 i$ & $7.2213+3.1650 i$ & $-1.6766+2.1050 i$ & $-1.5870-1.5825 i$ \\
\hline 0.202 & $-5.5875-4.1273 i$ & $6.6209+3.4331 i$ & $-1.6417+2.0637 i$ & $-1.5541-1.7166 i$ \\
\hline 0.240 & $-5.4402-4.0195 i$ & $6.1014+3.5850 i$ & $-1.6115+2.0098 i$ & $-1.4644-1.7925 i$ \\
\hline 0.440 & $-4.9159-3.4292 i$ & $3.7348+4.8267 i$ & $-1.4907+1.7146 i$ & $-0.5866-2.4133 i$ \\
\hline 0.640 & $-4.6317-3.0393 i$ & $0.8816+6.7486 i$ & $-1.3979+1.5196 i$ & $0.8464-3.3743 i$ \\
\hline 0.840 & $-4.4966-2.6815 i$ & $-3.643+11.193 i$ & $-1.3125+1.3407 i$ & $3.2215-5.5964 i$ \\
\hline 1.040 & $-4.4921-2.1974 i$ & $-11.832+18.837 i$ & $-1.2174+1.0987 i$ & $7.4949-9.4187 i$ \\
\hline 1.240 & $-4.6038-1.8406 i$ & $-27.338+33.651 i$ & $-1.1080+0.9203 i$ & $15.490-16.825 i$ \\
\hline 1.440 & $-4.8063-1.2685 i$ & $-58.743+61.022 i$ & $-0.9925+0.6342 i$ & $31.511-30.511 i$ \\
\hline
\end{tabular}




$$
\begin{aligned}
G_{T}^{(P)}\left(q^{2}\right) & =\left.\frac{i p \cdot Q}{m_{V}\left(m_{B}-m_{P}\right)} \frac{i f_{P}}{f_{V}^{\|}} G_{3}^{(\|)}\left(q^{2}\right)\right|_{\phi_{\| \rightarrow \phi_{P}}} \\
& =\left.\frac{-\left(m_{B}^{2}-q^{2}\right)}{2 m_{V}\left(m_{B}-m_{P}\right)} \frac{f_{P}}{f_{V}^{\|}} G_{3}^{(\|)}\left(q^{2}\right)\right|_{\phi_{\|} \rightarrow \phi_{P}} .
\end{aligned}
$$

Thus, the result of the longitudinal vector meson entirely follows from the pseudoscalar in the ultrarelativistic limit. Note that the sign of this relation changes when $\left(1+\gamma_{5}\right) \rightarrow$ $\left(1-\gamma_{5}\right)$ in $\mathcal{O}_{8}(2)$ which is reflected in Eq. (5) as well.

\section{3. $P$ (pseudoscalar)}

Analogous to (A9) we parametrize $g_{T}^{(P)}$ as follows:

$$
k_{G}^{-1} g_{T}^{(P)}\left(q^{2}\right)=\frac{\alpha_{s}}{4 \pi} C_{F}\left(-\frac{1}{2}\right) f_{P} m_{b}^{2} Q_{b} \int_{0}^{1} d u t_{H}^{(P)}(u) \phi_{P}(u) .
$$

The entire expression of $t_{H}^{(P)}(u)$ is rather bulky so we shall give only one coefficient for $t_{H}^{(P)}(u)$,
$c_{b}^{P}=\frac{4 q_{R} P^{2}\left(m_{b}^{4}+m_{b}^{2}\left(P^{2}-2 p_{B}^{2}+q^{2}\right)+p_{B}^{2}\left(p_{B}^{2}-P^{2}\right)\right)}{m_{b}\left(m_{B}-m_{P}\right) \bar{u}\left(P^{4}+2 P^{2} q^{2}+q^{2}\left(q^{2}-4 p_{B}^{2}\right)\right)}$,

which at least allows our results to be verified partially.

\section{APPENDIX B: PHOTON DISTRIBUTION AMPLITUDE CONTRIBUTIONS}

In this section we present a brief discussion of the contributions due to the photon distribution amplitude. The latter corresponds to the LD part of the photon whereas the photon of perturbation theory corresponds to the SD contribution. They can be separated in a transparent way by the background gauge field technique [39].

We present results for the on-shell photon of the two diagrams shown in Fig. 8 which constitute corrections to the correlation function in Eq. (9) and its diagrams should be added to the series in Fig. 2. Extending our notation to include the photon DA we obtain

$$
\begin{aligned}
G_{i}^{\left(\phi_{\gamma}\right),(\perp, \|)}\left(q^{2}\right)= & \frac{f_{K^{*}}^{(\perp \|)} \alpha_{s} m_{b} C_{F}}{f_{B} m_{B}^{2}} \int_{m_{b}^{2}}^{s_{0}} e^{{\left(\frac{m_{B}^{2}-s}{M^{2}}\right)}^{2}}\left(\chi_{s}\left(q^{2}\right)\langle\bar{s} s\rangle \int_{0}^{1} \phi_{\perp}\left(\frac{m_{b}^{2}-q^{2}}{s-q^{2}}\right) \phi_{\gamma}(v) \rho_{i}^{s(\perp, \|)}(s, v) d v\right. \\
& \left.+\chi_{q}\left(q^{2}\right)\langle\bar{q} q\rangle \int_{0}^{1} \phi_{\perp}(u) \phi_{\gamma}\left(\frac{-P^{2}+q^{2}+\Delta}{2 q^{2}}\right) \theta\left(m_{b}^{2}+P^{2}-s\right) \rho_{i}^{q(\perp, \|)}(s, u) d u\right) d s
\end{aligned}
$$

with $\Delta \equiv \sqrt{\left(P^{2}+q^{2}\right)^{2}-4 q^{2}\left(s-m_{b}^{2}\right)}, \Omega \equiv \Delta\left(P^{2}-q^{2}\right)(1-2 u)+\Delta$ and $\Sigma \equiv\left(m_{b}^{2}-q^{2}\right)\left(P^{2}-q^{2}\right)+\bar{v} q^{2}\left(s-q^{2}\right)$ and

$$
\begin{aligned}
& \rho_{1}^{q \perp}=\frac{\pi Q_{q}\left(-P^{2}+q^{2}+\Delta\right)}{6 \Omega}, \quad \rho_{1}^{s \perp}=\frac{\pi q^{2} Q_{b}}{6 \Sigma}, \quad \rho_{2}^{q \perp}=\frac{\pi Q_{q}\left(-\left(P^{2}\right)^{2}+P^{2} \Delta-q^{2}\left(q^{2}-2 s+\Delta\right)\right)}{6 P^{2} \Omega}, \\
& \rho_{2}^{s \perp}=\frac{\pi q^{2} Q_{b}\left(q^{2}-s\right)}{6 P^{2} \Sigma}, \quad \rho_{3}^{q \perp}=\frac{\pi Q_{q}\left(P^{2}+q^{2}-2 s+\Delta\right)}{6 \Omega}, \quad \rho_{3}^{s \perp}=-\frac{\pi Q_{b}\left(P^{2}+q^{2}-s\right)}{6 \Sigma}, \\
& \rho_{3}^{q \|}=\frac{-2 m_{b} m_{K^{*}} P^{2} \pi Q_{q}}{3 \Omega\left(m_{B}^{2}-q^{2}\right)}, \quad \rho_{3}^{s \|}=\frac{m_{b} m_{K^{*}} P^{2} \pi Q_{b}}{3 \Sigma\left(m_{B}^{2}-q^{2}\right)} .
\end{aligned}
$$
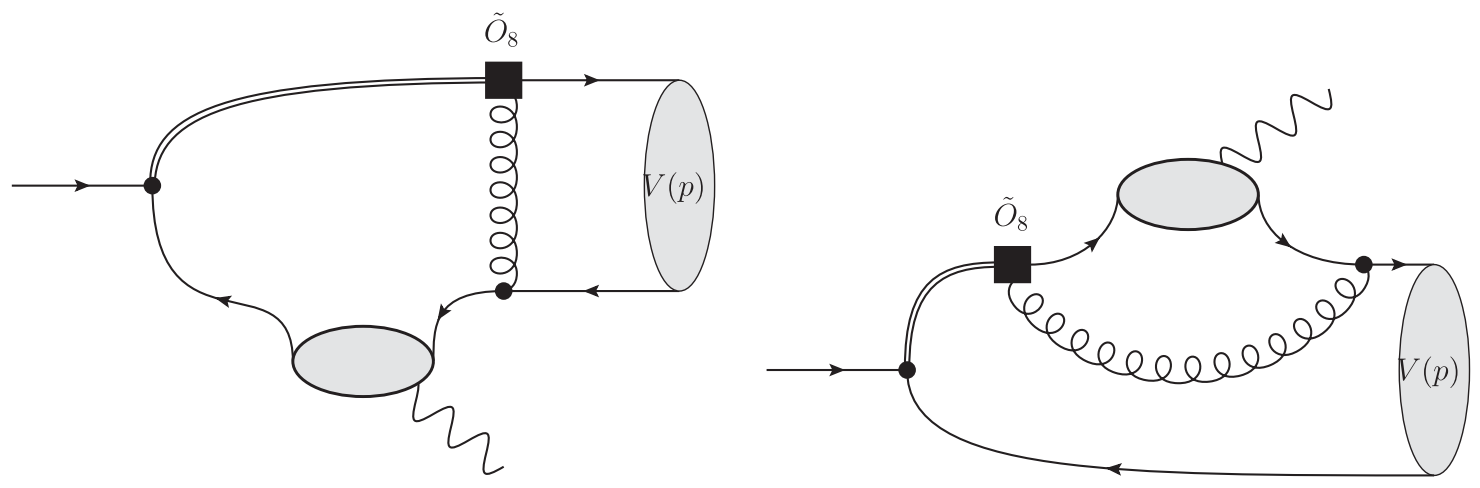

FIG. 8. Additional diagrams arising from the correlation function in Eq. (9). They correspond to the emission of a LD photon as described in the text. 
The definition of the leading twist-2 photon DA, denoted by $\phi_{\gamma}(u)$, can for example be found in Ref. [39]. Even though the photon DA is of twist-2 and suppressed with regard to the perturbative photon of twist- 1 , it is sometimes important because the photon susceptibility $\chi$, somewhat analogous to the light meson decay constants, turns out to be rather large, e.g., Ref. [39]. As it happens though all of the expressions above vanish for an on-shell photon $q^{2}=0$, except the $G_{3}$ part which does not contribute though to the rate at $q^{2}=0$. Presumably the vanishing of $G_{1,2}(0)^{\left(\phi_{\gamma}\right),(\perp, \|)}$ is accidental and higher twist photon DAs can be expected to contribute. One would expect the latter to be small though. The extension of the photon DA to off-shell photon $q^{2}>0$ has, to our knowledge, not been discussed systematically in the literature. One can get an idea of the size of the contributions by using the above computation with $q^{2}>0$ as well as $\chi\left(q^{2}\right)$ of Ref. [39] in Appendix B. The subscripts $q$ and $s$ for $\chi$ correspond to the susceptibility of $q=u, d$ and an $s$ flavor. We find that the contributions are around 5\% and thus fairly negligible in view of the overall uncertainty.

\section{APPENDIX C: HADRONIC INPUT VALUES}

The hadronic input for the vector DAs is summarized in Table V. For the pseudoscalar decay constants we take $f_{\pi}=0.131 \mathrm{GeV}$ and $f_{K}=0.160 \mathrm{GeV}$ [11] with negligible error and the data for the pseudoscalar meson DAs is taken from Ref. [41]:

$$
\begin{gathered}
a_{2}(\pi)=0.29(3)(7), \quad a_{2}(K)=0.24(3)(7), \\
a_{1}(K)=0.074(2)(4) .
\end{gathered}
$$

The latter value is in good agreement with Ref. [44].

The sum rule specific input can be found in Table VI. We assume $s_{0}\left[f_{H}\right]=s_{0}[H] \equiv s_{0}$ throughout. $s_{0}\left[B_{q}\right]=$ $35(1) \mathrm{GeV}^{2}$ is chosen as a reference value. All others are determined to satisfy $\left(m_{H_{q}}+X\right)^{2}=s_{0}\left[H_{q}\right]$ for

TABLE V. Note that $1^{--}$mesons with odd $G$-parity have vanishing odd Gegenbauer moments. The scale dependent quantities $f^{\perp}, a_{1,2}^{\|, \perp}$ are evaluated at $\mu=1 \mathrm{GeV}$. We use the updated value $\mathcal{B}\left(\tau \rightarrow K^{*} \nu_{\tau}\right)=1.20(7) \cdot 10^{-2}$ [11] as compared to the PDG value used by the end of $2006 \mathcal{B}\left(\tau \rightarrow K^{*} \nu_{\tau}\right)=1.29(5) \cdot 10^{-2}$ in Ref. [18], which leads to a decay constant which changes $f_{K^{*}}^{\|}$from 0.220 to $0.211 \mathrm{GeV}$ whereas all the others remain the same as in Ref. $[18]^{\mathrm{a}}$. The $f^{\perp}$ decay constants follow from the ratios $r[X]=f_{X}^{\perp}(2 \mathrm{GeV}) / f_{X}^{\|}$with $r[\rho]=$ $0.687(27), r\left[K^{*}\right]=0.712(12)$ and $r[\phi]=0.750(8)$ in Ref. [40]. Further, we use $r[\omega] \simeq r[\rho]$ in view of a lack of a lattice QCD determination of this quantity. For the DA parameters we have chosen to average $a_{1}^{\|}, a_{2}^{\|}\left(\rho, K^{*}, \phi\right)$ values from the lattice [41] with the sum rule determinations keeping the relative sum rule uncertainty, which is larger, in order to account for neglecting higher Gegenbauer moments. The references for the sum rule values are [42] for the $\rho$, [43] for the $\phi$ and $[44,45]$ for the $K^{*}$. In view of the lack of theoretical determinations of parameters for the $\omega$, we have assumed the same values as for the $\rho$ enlarging the uncertainty by a factor of 2 .

\begin{tabular}{lllllll}
\hline \hline & $f^{\|}[\mathrm{GeV}]$ & $f^{\perp}[\mathrm{GeV}]$ & \multicolumn{1}{c}{$a_{2}^{\|}$} & \multicolumn{1}{c}{$a_{2}^{\perp}$} & $a_{1}^{\|}$ & $a_{1}^{\perp}$ \\
\hline$\rho$ & $0.216(1)(6)$ & $0.160(11)$ & $0.17(7)$ & $0.14(6)$ & & \\
$\omega$ & $0.187(2)(10)$ & $0.139(18)$ & $0.15(12)$ & $0.14(12)$ & & \\
$K^{*}$ & $0.211(7)$ & $0.163(8)$ & $0.16(9)$ & $0.10(8)$ & $0.06(4)$ & $0.04(3)$ \\
$\phi$ & $0.235(5)$ & $0.191(6)$ & $0.23(8)$ & $0.14(7)$ & & \\
\hline \hline
\end{tabular}

${ }^{\mathrm{a}}$ With a numerical error corrected for $f_{\phi}^{\|}$as noted by the authors of Ref. [46].

TABLE VI. (left) $H$ stands for a heavy-light meson and $q$ stands for either a $u$ or $d$ quark. Sum rule specific values are in units of $\mathrm{GeV}$ to the appropriate power. $f_{H}$ correspond to the decay constants obtained from a tree-level sum rule. They should not be compared with the true value of $f_{H}$ as the latter have substantial radiative corrections in QCD sum rules. (middle) Condensates relevant for the $f_{H}$ sum rule (22). (right) Quark masses. The tree-level heavy quark masses are chosen to satisfy $m_{H} \simeq m_{h}+\bar{\Lambda}$ with $\bar{\Lambda} \simeq 0.6 \mathrm{GeV}$ approximately. The strange quark mass in the $\overline{\mathrm{MS}}$ corresponds to $\mu_{\overline{\mathrm{MS}}}=2 \mathrm{GeV}$. In the sum (22) $\bar{m}_{s}$ is scaled up to $\mu=\mu_{F}$.

\begin{tabular}{cccccc|cc|cc}
\hline \hline$H$ & $s_{0}$ & $M^{2}[G]$ & $M^{2}\left[f_{H}\right]$ & $m_{H}$ & $f_{H}(22)$ & Condensate & Value & Mass & Value \\
\hline$B_{s}$ & $36(1.5)$ & $9(2)$ & $5.0(5)$ & 5.37 & 0.162 & $\langle\bar{q} q\rangle$ & $(-0.24(1))^{3}$ & $m_{b}$ & $4.7(1)$ \\
$B_{q}$ & $35(1.5)$ & $9(2)$ & $5.0(5)$ & 5.28 & 0.142 & $\langle\bar{s} s\rangle$ & $0.8(1)\langle\bar{q} q\rangle$ & $m_{c}$ & $1.3(1)$ \\
$D_{s}$ & $6.7(7)$ & $6(2)$ & $1.5(2)$ & 1.96 & 0.185 & $\langle\bar{q} G q\rangle$ & $(0.8(1))^{2}\langle\bar{q} q\rangle$ & $\bar{m}_{s}$ & $0.094(3)$ \\
$D_{q}$ & $6.2(7)$ & $6(2)$ & $1.5(2)$ & 1.86 & 0.156 & $\langle\bar{s} G s\rangle$ & $(0.8(1))^{2}\langle\bar{s} s\rangle$ & & \\
\hline \hline
\end{tabular}


"universal" $X$. As discussed previously, $X$ is between the two pion mass and the $\rho$ threshold. The Borel parameter $M^{2}\left[f_{H}\right]$ of (22) is chosen in the minimum of the Borel window and in addition it is verified that the dimension five operators are below $10 \%$ and that the continuum contribution, vulnerable to quark-hadron duality violation, does not exceed $30 \%$. The Borel parameter $M^{2}[G]$ for the $G_{i}$ is chosen such that the continuum is $30 \%$; this choice suppresses higher twist corrections, which we have not computed, maximally.

\section{APPENDIX D: NONSPECTATOR CORRECTIONS $G^{(n s)}$}

The corrections which do not connect the gluon of the operator $\tilde{\mathcal{O}}_{8}$ with the spectator quark are depicted in Fig. 2 (bottom). They have been computed for the inclusive $b \rightarrow$ sll [16]. By gauge invariance the contribution is proportional to a function $F_{8}^{7(9)}\left(q^{2} / m_{b}^{2}\right)$ times the operator $\mathcal{O}_{7(9)}$. The latter reduces to the standard tensor and vector form factors $T_{i}\left(f_{T}\right)$ and $V, A_{i}\left(f_{+}\right)$when taken between $B$ and $V(P)$ states. We find

$$
\begin{aligned}
& G_{i}^{(\mathrm{ns})}\left(q^{2}\right)=\left(-\frac{\alpha_{s}\left(m_{b}\right)}{4 \pi}\right)\left(\frac{Q_{b}}{-1 / 3}\right)\left(F_{8}^{(7)} T_{i}\left(q^{2}\right)-F_{8}^{(9)} \frac{q^{2}}{2 m_{b}} V_{i}\left(q^{2}\right)\right), \quad i=1 \ldots 3, \\
& G_{T}^{(\mathrm{ns})}\left(q^{2}\right)=\left(-\frac{\alpha_{s}\left(m_{b}\right)}{4 \pi}\right)\left(\frac{Q_{b}}{-1 / 3}\right)(F_{8}^{(7)} f_{T}\left(q^{2}\right)-F_{8}^{(9)} \underbrace{\frac{q^{2}}{2 m b} v_{T}\left(q^{2}\right)}_{\frac{-m_{B}+m_{P}}{2 m_{b}} f_{+}\left(q^{2}\right)}),
\end{aligned}
$$

where $F_{8}^{7(9)}$ are given in Ref. [16] in terms of an expansion in powers of $q^{2} / m_{c}^{2}$ and a logarithm. The functions $\mathcal{V}_{i}$ and $v_{T}$ are defined as:

$\left\langle V(p, \eta)\left|\bar{s} \gamma^{\rho}\left(1-\gamma_{5}\right) b\right| \bar{B}\left(p_{B}\right)\right\rangle=P_{1}^{\rho} \mathcal{V}_{1}+P_{2}^{\rho} \mathcal{V}_{2}+P_{3}^{\rho} \mathcal{V}_{3}+\left[i\left(\eta^{*} \cdot q\right) q^{\rho}\right] \mathcal{V}_{\mathcal{P}}\left\langle P(p)\left|\bar{s} \gamma^{\rho} b\right| \bar{B}\left(p_{B}\right)\right\rangle=P_{T}^{\rho} v_{T}+q_{\rho} v_{S}$,

with

$$
\begin{aligned}
\mathcal{V}_{P} & =\frac{-2 m_{V}}{q^{2}} A_{0}\left(q^{2}\right) \quad \mathcal{V}_{1}=\frac{-V\left(q^{2}\right)}{m_{B}+m_{V}} \quad \mathcal{V}_{2}=\frac{-A_{1}\left(q^{2}\right)}{m_{B}-m_{V}} \quad \mathcal{V}_{3}=\left(\frac{m_{B}+m_{V}}{q^{2}} A_{1}\left(q^{2}\right)-\frac{m_{B}-m_{V}}{q^{2}} A_{2}\left(q^{2}\right)\right) \\
v_{s} & =\frac{m_{B}^{2}-m_{P}^{2}}{q^{2}} f_{0}\left(q^{2}\right) \quad v_{T}=-\frac{\left(m_{B}+m_{P}\right)}{q^{2}} f_{+}\left(q^{2}\right),
\end{aligned}
$$

where $V, A_{i}, f_{+}, f_{0}, f_{T}, T_{i}$ are all standard form factor notations in the literature. Note, as manifested by limiting the sum from $i=1 \ldots 3$, the $f_{0}\left(A_{0}\right)$ component does not contribute to $B \rightarrow V l l$ as the $q^{\rho}$ vanishes upon contraction with $\bar{l} \gamma_{\rho} l$ or the photon polarization tensor $\epsilon(q)$.

\section{APPENDIX E: LORENTZ STRUCTURES}

The Lorentz structures of the vector meson are given $b^{32}$

$$
\begin{aligned}
& P_{1}^{\rho}=2 \epsilon_{\alpha \beta \gamma}^{\rho} \eta^{* \alpha} p^{\beta} q^{\gamma} \\
& P_{2}^{\rho}=i\left\{\left(m_{B}^{2}-m_{V}^{2}\right) \eta^{* \rho}-\left(\eta^{*} \cdot q\right)\left(p+p_{B}\right)^{\rho}\right\} \\
& P_{3}^{\rho}=i\left(\eta^{*} \cdot q\right)\left\{q^{\rho}-\frac{q^{2}}{m_{B}^{2}-m_{V}^{2}}\left(p+p_{B}\right)^{\rho}\right\},
\end{aligned}
$$

and the one for the pseudoscalar meson is

$$
P_{T}^{\rho}=\frac{1}{m_{B}+m_{P}}\left\{\left(m_{B}^{2}-m_{P}^{2}\right) q^{\rho}-q^{2}\left(p+p_{B}\right)^{\rho}\right\} .
$$

All projectors are transverse, i.e., $q \cdot P=0$ when on-shell momentum relations like $p_{B}^{2}=m_{B}^{2}$ etc. are taken into

\footnotetext{
${ }^{32}$ The sign convention for the epsilon tensor is given by $\operatorname{tr}\left[\gamma_{5} \gamma_{a} \gamma_{b} \gamma_{c} \gamma_{d}\right]=4 i \epsilon_{a b c d}$ and the conventions we use are those used in the classic textbook of Bjorken and Drell.
}

account. The structure $P_{3}=P_{3}^{\rho} \epsilon(q)_{\rho}$ is absent for an on-shell photon since $\left.\epsilon(q) \cdot P_{3}\right|_{q^{2}=0}=0$ and thus $P_{3}$ can be seen as a purely longitudinal part of the photon. Note that $P_{3}^{\rho}=i /\left.\left(m_{B}-m_{P}\right)\left(\eta^{*} \cdot q\right) P_{T}^{\rho}\right|_{m_{P} \rightarrow m_{V}}$.

\section{Extension to include spurious momentum}

The extension of the Lorentz structures to include the spurious momentum $k$ in the vector case (E1) is

$$
\begin{aligned}
\left(p_{1}\right)_{\rho} & =2 \epsilon_{\alpha \beta \gamma}^{\rho} \eta^{* \alpha} p^{\beta} Q^{\gamma} \\
\left(p_{2}\right)_{\rho} & =i\left[\left(\left(p_{B}+p\right) \cdot Q\right) \eta_{\rho}^{*}-\left(\eta^{*} \cdot Q\right)\left(p_{B}+p\right)_{\rho}\right] \\
\left(p_{3}\right)_{\rho} & =i\left[\left(\eta^{*} \cdot Q\right) Q_{\rho}-\left(\eta^{*} \cdot Q\right)\left(p_{B}+p\right)_{\rho} \frac{q^{2}}{Q \cdot\left(p_{B}+p\right)}\right] \\
\left(p_{4}\right)_{\rho} & =i\left[\left(\eta^{*} \cdot Q\right) k_{\rho}-\left(\eta^{*} \cdot Q\right)\left(p_{B}+p\right)_{\rho} \frac{k \cdot Q}{Q \cdot\left(p_{B}+p\right)}\right]
\end{aligned}
$$

and in the pseudoscalar case (E2) is

$$
\begin{aligned}
& \left(p_{T}\right)_{\rho}=\left(m_{B}-m_{P}\right)\left[Q_{\rho}-\frac{q^{2}}{Q \cdot\left(p_{B}+p\right)}\left(p_{B}+p\right)_{\rho}\right] \\
& \left(\bar{p}_{\bar{T}}\right)_{\rho}=\left(m_{B}-m_{P}\right)\left[k_{\rho}-\frac{k \cdot Q}{Q \cdot\left(p_{B}+p\right)}\left(p_{B}+p\right)_{\rho}\right] .
\end{aligned}
$$


Essentially, we get one more structure due to a linearly independent vector $k$ and the projectors are extended such that they remain transverse, i.e., $Q \cdot q=0$. This is easy to verify using $q^{2}=Q^{2}$. Since $p_{3}^{\rho}=(\eta \cdot Q) p_{T}^{\rho}$ we have

$$
p_{3}^{\rho} \rightarrow\left(\frac{i p \cdot Q}{m_{V}\left(m_{B}-m_{V}\right)}\right) p_{T}^{\rho}=\left(\frac{i\left(P^{2}-q^{2}\right)}{2 m_{V}\left(m_{B}-m_{V}\right)}\right) p_{T}^{\rho}
$$

in the ultrarelativistic limit $\eta \rightarrow p / m_{V}$ as discussed in Appendix A 2. In the last equality we have used the approximation $p^{2}=0$.

\section{APPENDIX F: DISTRIBUTION AMPLITUDES}

The leading twist (twist-2) DAs for the pseudoscalar (e.g., Ref. [20]) and vector (e.g., Ref. [28]) mesons are defined as follows:

$$
\begin{aligned}
\left\langle K(p)\left|[\bar{s}(x)]_{\alpha} \ldots[q(z)]_{\beta}\right| 0\right\rangle= & i \frac{f_{K}}{4}\left[\not p \gamma_{5}\right]_{\beta \alpha} \int_{0}^{1} d u e^{i u x \cdot p+i \bar{u} z \cdot p} \phi_{K}(u)+\ldots \\
\left\langle K^{*}(p, \eta)\left|[\bar{s}(x)]_{\alpha} \ldots[q(z)]_{\beta}\right| 0\right\rangle= & \frac{f_{K^{*}}^{\perp}}{4}\left[\eta^{*}(p) \not p\right]_{\beta \alpha} \int_{0}^{1} d u e^{i u x \cdot p+i \bar{u} z \cdot p} \phi_{\perp}(u) \\
& +m_{K^{*}} \frac{f_{K^{*}}}{4}[\not p]_{\beta \alpha} \frac{\eta^{*} \cdot(x-z)}{p \cdot(x-z)} \int_{0}^{1} d u e^{i u x \cdot p+i \bar{u} z \cdot p} \phi_{\|}(u)+\cdots,
\end{aligned}
$$

which we have chosen to be represented by the kaons for definiteness.

\section{APPENDIX G: CONTACT TERMS AND WARD-TAKAHASHI IDENTITIES}

The aim of this Appendix is to clarify the issue of nontransverse terms in the correlation function $\Pi_{\rho}^{P, V}(9)$. Let us make two points before we draw the conclusion for the significance of the computation of the $G_{\iota}$ functions.

(1) We would like to observe that the matrix elements $\mathcal{A}^{* \rho}(P, V)$ are transverse, i.e., $q_{\rho} \mathcal{A}^{* \rho}(P, V)=0$, by virtue of conservation of the electromagnetic current $\partial \cdot j^{\mathrm{em}}=0$ or gauge invariance. The statement is even true for off-shell photons $q^{2} \neq 0$ for the SD part defined by a current insertion as in Eq. (3). This is readily derived by integration by parts, e.g., Ref. [12]. Thus we were right to use transverse projectors only.

(2) More complicated cases arise from contact terms due to charged operator insertions on the level of the correlation function $\Pi_{\rho}^{P, V}(9)$. This is formalized in terms of a WTI for the correlation function, which we have used as a check of our computation. Consider the correlation function, as depicted in Fig. 9,

$$
\begin{aligned}
C_{\rho}= & i \int_{x, y, z} e^{-i p_{B} \cdot x+i Q \cdot y+i u x_{1} \cdot p+i \bar{u} x_{2} \cdot p} \\
& \times\langle 0| T J_{B}(x) j_{\rho}^{\mathrm{em}}(y) \bar{q} \not A q(z) \bar{s}\left(x_{1}\right) u \not p \\
& \times \mathcal{P} \bar{u} \not p q\left(x_{2}\right) \tilde{O}_{8}(0)|0\rangle,
\end{aligned}
$$

with an unspecified projector $\mathcal{P}$. Note that one could equally well leave the two open indices instead of inserting $\mathcal{P}$. This correlation function corresponds to the one we use in our computation modulo the convolution and the specific projection $\mathcal{P}$ of the DA. The WTI specifies what happens under contraction with $Q_{\rho}$ :

$$
Q^{\rho} C_{\rho}=3 \text { contact term in Fig. } 10 .
$$

We have verified in each case that this identity is satisfied for unspecified $\mathcal{P}$. The contact terms arise when the derivative acts on the $T$ product and gives rise to $\left[j_{0}, \mathcal{O}\right]=q_{\mathcal{O}} \mathcal{O}$ type terms, e.g., Ref. [12], where $q_{\mathcal{O}}$ is the charge of the operator $\mathcal{O}$. The three contact terms, corresponding to the charged operators, are depicted in Fig. 10.

The question that imposes itself is, how can transversity of the amplitude and the nontransversity of the correlation $C_{\rho}$, used to extract the $G_{\iota}$ functions, be reconciled? One might think that the contact terms disappear once we go "on shell," by which we mean specifying the projector to be

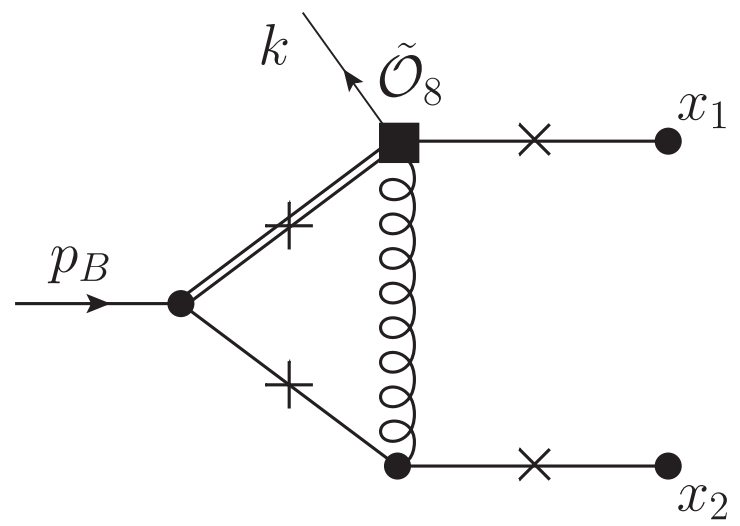

FIG. 9. Correlation function $C^{\rho}$ in Eq. (G1). The crosses denote the four possible places where the perturbative photon of momentum $Q$ can be radiated from. 

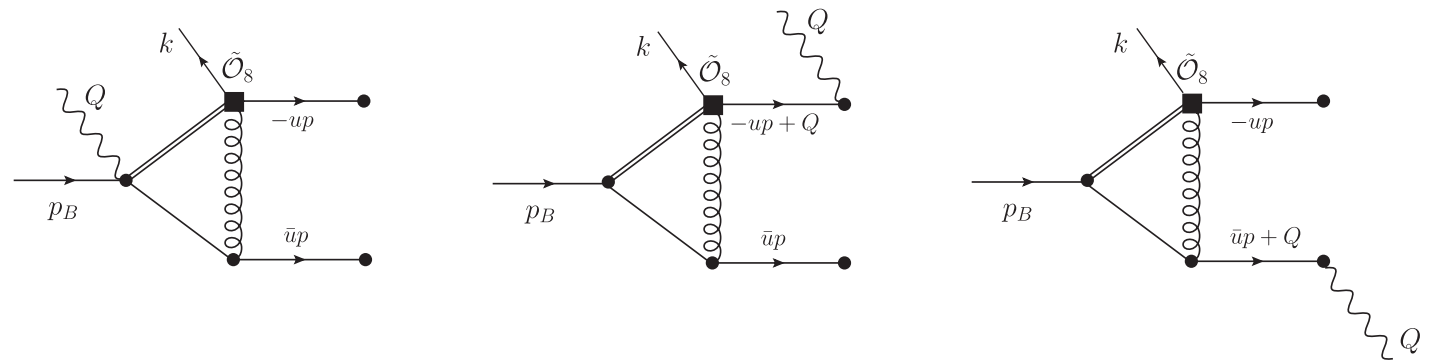

FIG. 10. Contact terms for the "off-shell" WTI. The diagram on the left is proportional to the charge of the $B$ meson whereas the middle and right diagram are proportional to the charge of the $s$ quark and the spectator quark respectively. Only the diagram on the left needs to be computed anew; the other two diagrams are proportional to $\left.u p \cdot \mathcal{A}\right|_{A_{4}}$ and $\left.\bar{u} p \cdot \mathcal{A}\right|_{A_{1}}$ respectively.

$\mathcal{P} \sim\left(\not p \gamma_{5}, \not p,[\not p \eta]\right)$ for the DA $\left(\phi_{P}, \phi_{\perp}, \phi_{\|}\right)$respectively. Nontransverse structures remain for $P / V_{\|}$but not for $V_{\perp}$; $g_{0}^{(P)} \sim g_{0}^{(\|)} \neq 0$ and $g_{0}^{(\perp)}=0$; cf. Eq. (A2) for the latter. It is the diagram to the left, in which the photon is radiated from the charged $J_{B^{-}}$, that gives a nonvanishing contribution. The momentum flowing into this vertex is $\left(p_{B}-Q\right)^{2}=$ $(p+k)^{2}=p_{B}^{2}-P^{2}$. The transverse part is proportional to PV functions of the type $B_{0}\left(p_{B}^{2}-P^{2}, 0, m_{b}^{2}\right)$ as expected and displays a cut in $p_{B}^{2}>m_{b}^{2}+P^{2}=m_{b}^{2}+m_{B}^{2}$. This contribution can be seen as yet another parasitic cut. It is of no relevance though in the final dispersion integral in $p_{B}^{2}$ since the threshold is well above the continuum threshold $s_{H} \simeq s_{0}$ in relations like (15) and (17).

\section{APPENDIX H: ANALYTIC STRUCTURE AND DISPERSION REPRESENTATION}

Let us parametrize a dispersion representation as follows:

$$
f\left(p_{B}^{2}\right)=\int_{0}^{\infty} \frac{\rho_{f}}{s-p_{B}^{2}-i 0}+\left[f\left(p_{B}^{2}\right)\right]_{A n}+\text { subtractions. }
$$

The polynomial subtraction terms, as previously emphasized, are of no importance as they vanish under the Borel transformation. The term $[f]_{A n}$ corresponds to an anomalous threshold. Amongst the PV functions (A1) present in the results, given in Appendix A, solely $C_{a}{ }^{33}$ includes an anomalous threshold which extends into the lower complex half-plane (cf. Fig. 11) at physical momenta $P^{2}, q^{2}>0$. This is discussed in Sec. $\mathrm{H} 1$ from various viewpoints. In addition, the density $\rho_{C_{a}}$ necessitates many case distinctions, which is not uncommon for a vertex function, e.g., Ref. [47].

We have checked the dispersion relations by comparing them against LoopTools [48] which allows for numerical evaluation of the scalar PV functions. Below, we shall quote the results, starting with the anomalous part of $C_{a}$ :

$$
\left[C_{a}\left(p_{B}^{2}\right)\right]_{A n}=-2 \pi i \int_{s_{+}}^{\mathrm{Re} s_{+}} \frac{d s}{s-p_{B}^{2}} \frac{1}{\sqrt{\lambda}}
$$

$s_{+}$is one of the two solutions of the leading Landau equations of the graph

$$
s_{ \pm}=\frac{(1+u) m_{b}^{2}+u P^{2} \pm \sqrt{\left(u P^{2}-\bar{u} m_{b}^{2}\right)^{2}-4 u^{2} m_{b}^{2} q^{2}-i 0}}{2 u},
$$

where the $-i 0$ implies that $\operatorname{Im} s_{+} \leq 0$. The densities $\rho_{f}$ of the representation $(\mathrm{H} 1)$ are

$$
\begin{aligned}
\rho_{B_{a}}= & \left(1-\frac{m_{b}^{2}}{u\left(s-P^{2}\right)}\right) \Theta\left(s-\frac{m_{b}^{2}}{u}-P^{2}\right) \\
\rho_{B_{c}}= & \left(1-\frac{m_{b}^{2}}{u s+\bar{u} q^{2}}\right) \Theta\left(s-\frac{m_{b}^{2}-\bar{u} q^{2}}{u}\right) \\
\rho_{C_{a}}= & \left(\frac{\operatorname{Im}\left[C_{a}\right]}{\pi}+\frac{1}{\sqrt{\lambda}}\left(\log _{L}\left(\frac{z_{+}-z_{L}}{z_{-}-z_{L}}\right)-\log \left(\frac{z_{+}-1}{z_{-}-1}\right)\right)\right) \Theta\left(s-m_{b}^{2}\right) \\
\rho_{C_{c}}= & \frac{\log \left(\frac{A-\sqrt{\lambda_{1} \lambda_{3}}}{A+\sqrt{\lambda_{1} \lambda_{3}}}\right)}{\sqrt{\lambda_{3}}}\left[\Theta\left(s-\frac{m_{b}^{2}-\bar{u} q^{2}}{u}\right)-\Theta\left(s-\frac{m_{b}^{2}}{u}-P^{2}\right)\right]+\frac{\log \left(\left(\frac{B-\sqrt{\lambda_{2} \lambda_{3}}}{B+\sqrt{\lambda_{2} \lambda_{3}}}\right)\left(\frac{A-\sqrt{\lambda_{1} \lambda_{3}}}{A+\sqrt{\lambda_{1} \lambda_{3}}}\right)\right)}{\sqrt{\lambda_{3}}} \Theta\left(s-\frac{m_{b}^{2}}{u}-P^{2}\right),
\end{aligned}
$$

where

\footnotetext{
${ }^{33} C_{b}$ corresponds to $\left.C_{a}\right|_{u \rightarrow 1}$ and so we shall not discuss it separately as well as all other functions on the rhs of the list in Eq. (A1)
} 

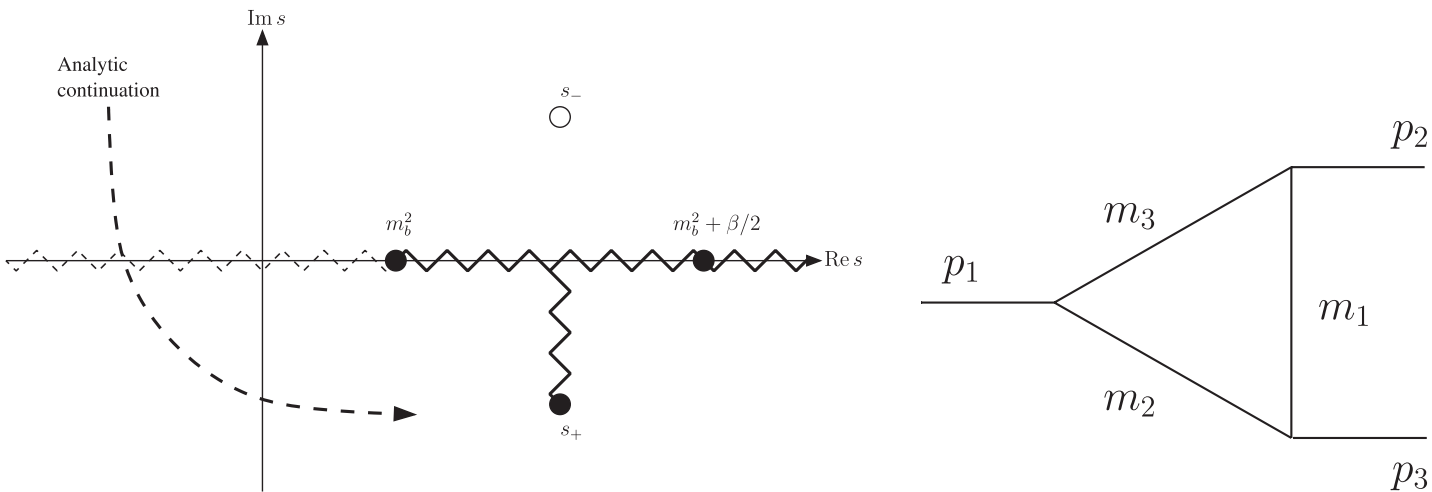

FIG. 11. Analytic structure of $C_{0}\left(s, s-\beta, \alpha, 0, m_{b}^{2}, 0\right)$. The path of the branch cut connected to the branch point $s_{+}$can be inferred from a deformation analysis as in Ref. [49]. (left) Black spots correspond to branch points on the physical sheet. The white spot branch point is not on the physical sheet. Black zigzag lines are branch cuts on the physical sheet. The dashed zigzag line corresponds to a branch cut of $C_{a}^{F}$ (H17) but not of $C_{a}=C_{0}\left(s, s-\beta, \alpha, 0, m_{b}^{2}, 0\right)$ as explained in the text. The arrow indicates around which branch point $C_{a}^{F}$ is analytically continued into the lower half-plane. (right) Triangle graph corresponding to the $C_{0}\left(p_{1}^{2}, p_{2}^{2}, p_{3}^{2}, m_{2}^{2}, m_{3}^{2}, m_{1}^{2}\right) \mathrm{PV}$ function. The conventions are the same as in LoopTools [48] and FeynCalc [22].

$$
\begin{aligned}
A & \equiv 2 m_{b}^{2} q^{2}-u\left(q^{2}-P^{2}\right)\left(m_{b}^{2}+\bar{u} q^{2}+u s\right) & B & \equiv u\left(\left(q^{2}-P^{2}\right)\left(m_{b}^{2}+u\left(s-P^{2}\right)\right)-2 q^{2}\left(s-P^{2}\right)\right) \\
\lambda_{1} & \equiv \lambda\left(u s+\bar{u} q^{2}, m_{b}^{2}, 0\right), & \lambda_{2} & \equiv \lambda\left(u\left(s-P^{2}\right), m_{b}^{2}, 0\right), \\
\lambda_{3} & \equiv \lambda\left(u s+\bar{u} q^{2}, u\left(s-P^{2}\right), q^{2}\right), & \lambda & \equiv \lambda\left(p_{B}^{2}, \bar{u} P^{2}+u q^{2}, u\left(p_{B}^{2}-P^{2}\right)\right)
\end{aligned}
$$

and $\lambda(x, y, z)=(x-(y+z))^{2}-4 y z$ is the Källén function.

The notation $\log _{-}$and $\log _{L}$ in the density $\rho_{C_{a}}$ demands clarification:

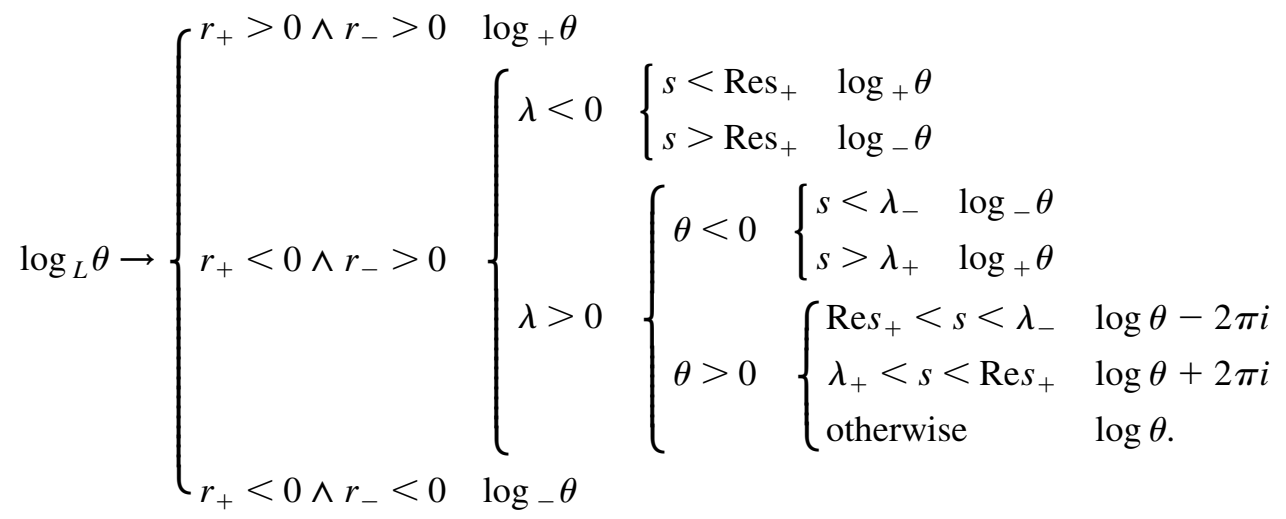

The square root of $\lambda$, but not $\lambda_{1,2,3}$, in Eq. (H4) is to be taken as

$$
\sqrt{\lambda} \rightarrow \begin{cases}\sqrt{\lambda} & s<\lambda_{-} \\ i \sqrt{-\lambda} & \lambda_{-}<s<\lambda_{+} \\ -\sqrt{\lambda} & s>\lambda_{+} .\end{cases}
$$

Furthermore, $\log _{ \pm}$are defined as follows:

$$
\begin{aligned}
& \log _{+} x= \begin{cases}\log x & \operatorname{Im} x=0 \\
\log (-x)+i \pi & \operatorname{Im} x \neq 0\end{cases} \\
& \log _{-} x=\log (-x)-i \pi
\end{aligned}
$$

The remaining variables in $\rho_{C_{a}}$ are given by 


$$
\begin{aligned}
\lambda_{ \pm} & =\frac{\bar{u} P^{2}+u(1+u) q^{2} \pm 2 u \sqrt{q^{2}\left(\bar{u} P^{2}+u q^{2}\right)}}{\bar{u}^{2}}, \\
\lambda & =\bar{u}^{2}\left(s-\lambda_{+}\right)\left(s-\lambda_{-}\right) \\
z_{ \pm} & =\frac{(1+u) p_{B}^{2}-P^{2}-u q^{2} \pm \sqrt{\lambda}}{2 p_{B}^{2}}, \\
z_{L} & =1+\frac{\bar{u} P^{2}+u q^{2}}{m_{b}^{2}-p_{B}^{2}} \quad r_{ \pm}=r\left(\lambda_{ \pm}\right), \\
r\left(p_{B}^{2}\right) & =\left(1+u-2 z_{L}\right) p_{B}^{2}-P^{2}-u q^{2} .
\end{aligned}
$$

\section{Analytic structure of $C_{0}\left(s, s-\beta, \alpha, 0, m_{b}^{2}, 0\right)$ in $\mathbb{C}_{s}$}

In this section we shall discuss the analytic properties of the PV function $C_{a}$ through a function with simplified but equivalent variables, namely,

$$
C_{0}\left(s, s-\beta, \alpha, 0, m_{b}^{2}, 0\right)
$$

with conventions as indicated in the caption of Fig. 11 (right). The function (H11) corresponds to $C_{a}$ in Eq. (A1) with the following substitutions:

$$
s=p_{B}^{2}, \quad \alpha=u q^{2}+\bar{u} P^{2}, \quad \beta=u P^{2}+\bar{u} s .
$$

It is argued in a succession of rigor, first from the viewpoint of Landau equations $\mathrm{H} 1 \mathrm{a}$, then explicit one-loop solutions and uniqueness of analytic continuation ( $\mathrm{H} 1 \mathrm{~b})$ and finally axiomatic results by Källén and Wightman (H $1 \mathrm{c}$ ), that the correlation function has a complex anomalous threshold on the physical sheet for

$$
\alpha>\alpha^{*} \equiv \frac{\beta^{2}}{4 m_{b}^{2}} .
$$

\section{a. Singularities from the Landau equations}

The Landau equations $[14,15]$ are a means to determine singularities of a perturbative diagram. ${ }^{34}$ The crucial and limiting point is that, unless the singularities are real, there is no direct way to determine on which Riemann sheets they appear.

We shall be interested in determining the so-called leading Landau singularity of the triangle graph [Fig. 11 (right)], also known as an anomalous threshold. It corresponds to all three propagators being on shell. The condition can conveniently be written in terms of a determinant,

\footnotetext{
${ }^{34}$ Singularities which arise due to infinite loop momentum are possible to interpret through the Landau equations (though not easily) and have therefore been called singularities of the second type or non-Landau singularities.
}

$$
\begin{gathered}
\operatorname{det}\left(\begin{array}{ccc}
1 & x_{1} & x_{2} \\
x_{1} & 1 & x_{3} \\
x_{2} & x_{3} & 1
\end{array}\right)=0, \quad x_{i} \equiv \frac{p_{i}^{2}-m_{j}^{2}-m_{k}^{2}}{2 m_{j} m_{k}}, \\
i \neq j \neq k \neq i,
\end{gathered}
$$

where $m_{j}$ and $m_{k}$ are the masses of the propagators adjacent to the ingoing momentum squares $p_{i}^{2}$. For the $C_{0}$ in question (H11), this leads to the Landau surface

$$
\left(s-m_{b}^{2}\right)\left(s-m_{b}^{2}-\beta\right)+\alpha m_{b}^{2}=0
$$

whose solutions are given by

$$
s_{ \pm}=m_{b}^{2}+\beta / 2 \pm \sqrt{(\beta / 2)^{2}-\alpha m_{b}^{2}}
$$

As long as $\alpha<\alpha^{*}$ (H13) the solutions are real and we can decide whether they are on the physical sheet or not by checking whether the Landau equations admit solutions where the Feynman parameter admits values between $[0,1]$. As a matter of fact for any $q^{2}>0$ [cf. Eq. (H12)], there exists some $u \in[0,1]$ for which $\alpha>\alpha^{*}$. Thus we are led to the question of whether or not the singularities $s_{ \pm}$ are on the physical sheet. Some guidance can be gained following Mandelstam contour deformation prescription [49]. The idea is that one starts with values for $P^{2}$ and $Q^{2}$ such that $s_{ \pm}$are real. Then a dispersion representation can be constructed by checking which singularities are on the physical sheet. Upon deformation of the external momenta $\left(P^{2}, Q^{2}\right)$ the contour is deformed such that no singularities are crossed. Applying this procedure we found that $s_{+}$is on the physical sheet and $s_{-}$on an unphysical sheet. In the next section we shall show the same result to be true in a more explicit and possibly more transparent way from the known one-loop result.

\section{b. Complex branch points in the lower half-plane from analytic continuation of the Feynman parameter representation}

Here we discuss the function $C_{a}$ (A1) itself rather than $C_{0}$ (H11) because reference is made to the variables used in $\rho_{C_{a}}$ (H4) and thereafter. Variables are restricted to the following values: $0 \leq u \leq 1, m_{B}^{2}>m_{b}^{2}>0, P^{2}=m_{B}^{2}+i 0$ and $q^{2}-i 0=\operatorname{Re}\left[q^{2}\right]>0$. Our two main ingredients are the uniqueness of analytic continuation from the real line and the fact that the lowest cut on the real line starts at $m_{b}^{2}$. The latter can be verified from the Landau equations.

The correlation function $C_{a}$, originally defined just above the real line of $p_{B}^{2}\left(\right.$ at $\left.\operatorname{Re}\left[p_{B}^{2}\right]+i 0\right)$, can be analytically continued into the entire upper half-plane by the Feynman parameter integral representation, 


$$
C_{a}^{F}\left(p_{B}^{2}\right)=\int_{0}^{1} d x \int_{0}^{1-x} d y\left[(1-x-y)\left(x p_{B}^{2}+y u\left(p_{B}^{2}-P^{2}\right)-m_{b}^{2}\right)+x y\left(\bar{u} P^{2}+u q^{2}\right)+i 0\right]^{-1},
$$

since it is free from singularities in this region. For $\operatorname{Im}\left[p_{B}^{2}\right] \neq 0$ (where the $i 0$ prescription is irrelevant) $C_{a}^{F}\left(p_{B}^{2 *}\right)=C_{a}^{F}\left(p_{B}^{2}\right)^{*}$ by inspection. This implies that $C_{a}^{F}$, but not necessarily $C_{a}$, has got a branch cut on the real axis whenever $\operatorname{Im}\left[C_{a}^{F}\left(p_{B}^{2}\right)\right] \neq 0$. Note these are the only possible singularities for the range of variables mentioned above.

Using the Feynman parameter representation $C_{a}^{F}\left(p_{B}^{2}\right)$ as a starting point we construct an analytic continuation to the lower half-plane as follows:

$C_{a}\left(p_{B}^{2}\right)= \begin{cases}C_{a}^{F}\left(p_{B}^{2}\right) & \operatorname{Im}\left[p_{B}^{2}\right]>0 \\ C_{a}^{F}\left(p_{B}^{2 *}\right)^{*}+C_{a}^{\mathrm{rem}}\left(p_{B}^{2}\right) & \operatorname{Im}\left[p_{B}^{2}\right]<0 .\end{cases}$

The reminder function $C_{a}^{\mathrm{rem}}\left(p_{B}^{2}\right)$ is defined such that there is no branch cut below $p_{B}^{2}<m_{b}^{2}$ for $C_{a}\left(p_{B}^{2}\right)$. To remove the branch cut near a given $p_{B}^{2}$ we require that $C_{a}\left(p_{B}^{2}\right)$ in (H18) is equal immediately above and below the real line which enforces

$$
C_{a}^{\mathrm{rem}}\left(p_{B}^{2}\right)=2 i \operatorname{Im}\left[C_{a}^{F}\left(p_{B}^{2}\right)\right], \quad \operatorname{Im}\left[p_{B}^{2}\right]=0 .
$$

The resulting function eliminates the branch cut for $p_{B}^{2}<m_{b}^{2}$. In this region a remainder function $C_{a}^{\mathrm{rem}}\left(p_{B}^{2}\right)$ may be derived from (H19) and (H17) using $1 /(x+i 0)=\operatorname{PP}[1 / x]-i \pi \delta(x)^{35}$ to give

$C_{a}^{\mathrm{rem}}\left(p_{B}^{2}\right)=-\frac{2 \pi i}{\sqrt{\lambda}}\left(\log \left(\frac{z_{+}-z_{L}}{z_{+}-1}\right)-\log \left(\frac{z_{-}-z_{L}}{z_{-}-1}\right)\right)$,

with $z_{ \pm}, z_{L}$ and $\lambda$ as in (H10). ${ }^{36}$ The branch points of the logarithms and square roots appear on all Riemann sheets unless there are cancellations between terms.

\footnotetext{
${ }^{36}$ Note that while the directions of the cuts are ambiguous the branch points $s_{ \pm}$are unambiguous. Fortunately it is the latter we are interested in. In other words, the exact location of the cuts is somewhat analogous to the choice of a coordinate system whereas the branch points are not dependent on it.

${ }^{37}$ Note that while the directions of the cuts are ambiguous the branch points $s_{ \pm}$are unambiguous. Fortunately it is the latter we are interested in. In other words, the exact location of the cuts is somewhat analogous to the choice of a coordinate system whereas the branch points are not dependent on it.
}

The branch cuts of the two logarithms start at $z_{ \pm}=z_{L}$ (there are no solutions for $\left|p_{B}^{2}\right|<\infty$ to $z_{ \pm}=1$ ), which occurs at $p_{B}^{2}=s_{ \pm}$, and since the branch points $s_{ \pm}$are separate no cancellation occurs and there indeed must be a cut on all Riemann sheets of $C_{a}^{\mathrm{rem}}\left(p_{B}^{2}\right)$. $s_{ \pm}$is complex for physical momenta, and since we know that $C_{a}^{\mathrm{rem}}\left(p_{B}^{2}\right)$ is the only term with branch points away from the real line in (H18) we conclude that analytically continuing (H17) to $\operatorname{Im}\left[p_{B}^{2}\right]<0$ across the real line, to the left of the branch point $p_{B}^{2}=m_{b}^{2}$ [cf. Fig. 11 (left)], necessarily results in a branch cut off the real line in the lower complex half-plane. To this end we note that $C_{a}^{\mathrm{rem}}\left(p_{B}^{2}\right)$ corresponds to $\rho_{C_{a}}(\mathrm{H} 4)$ modulo the imaginary part.

In the next section we are going to learn that the complex singularities are not an artifact of perturbation theory but are expected on most general grounds from axiomatic approaches.

\section{c. The Källén-Wightman domain}

Based on axioms such as Lorentz covariance, assumptions on the spectrum and microcausality Källén and Wightman [50] obtained results on the domain analyticity of the vacuum expectation value of three scalar fields. We note that the $C_{0} \mathrm{PV}$ function is simply a one-loop approximation in a specific theory with three point interactions. Denoting the three invariant momentum squares of the three vertices by $Z_{i}=p_{i}^{2}$, for $i=1 \ldots 3$, the domain can be separated into eight regions characterized by the signs of $\operatorname{Im}\left[Z_{i}\right]$, denoted by $[ \pm \pm \pm]$. Those eight octants are partly separated by the normal cuts. In addition the domains with signatures $[++-]$ and $[--+]$ and permutations thereof have the following boundaries [51]:

$$
\left(Z_{1}-r\right)\left(Z_{2}-r\right)+r Z_{3}=0, \quad r>0,
$$

with $\operatorname{Im}\left(Z_{1}\right) \operatorname{Im}\left(Z_{2}\right)>0$. Thus for $\left(Z_{1}, Z_{2}, Z_{3}\right)=$ $(s, s-\beta, \alpha+i 0)$ with $\operatorname{Im}[s]<0$ we find

$$
(s-r)(s-\beta-r)+r \alpha=0,
$$

which corresponds to the Landau surface equation (H15) upon identifying $r=m_{b}^{2}$.
[1] P. Colangelo and A. Khodjamirian, in At the Frontier of Particle Physics, edited by M. Shifman (World Scientific, Singapore, 2001), Vol. 3, pp. 1495-1576.
[2] S. W. Bosch and G. Buchalla, Nucl. Phys. B621, 459 (2002).

[3] M. Beneke, T. Feldmann, and D. Seidel, Nucl. Phys. B612, 25 (2001). 
[4] A. L. Kagan and M. Neubert, Phys. Lett. B 539, 227 (2002).

[5] T. Feldmann and J. Matias, J. High Energy Phys. 01 (2003) 074.

[6] Y. Y. Keum, M. Matsumori, and A. I. Sanda, Phys. Rev. D 72, 014013 (2005).

[7] A. Khodjamirian, T. Mannel, and Y.-M. Wang, arXiv:1211.0234 [J. High Energy Phys. (to be published)].

[8] G. Isidori and J. F. Kamenik, Phys. Rev. Lett. 109, 171801 (2012).

[9] J. Lyon and R. Zwicky, arXiv:1210.6546.

[10] M. A. Shifman, A. I. Vainshtein, and V. I. Zakharov, Nucl. Phys. B147, 385 (1979).

[11] J. Beringer et al. (Particle Data Group Collaboration), Phys. Rev. D 86, 010001 (2012).

[12] S. Weinberg, Foundations, The Quantum Theory of Fields (Cambridge University Press, Cambridge, England, 1995), Vol. 1, p. 609.

[13] G. Källén, Helv. Phys. Acta 25, 417 (1952); H. Lehmann, Nuovo Cimento 11, 342 (1954).

[14] R. J. Eden, P. V. Landshoff, D. I. Olive, and J.C. Polkinghorne, The Analytic S-Matrix (Cambridge University Press, Cambridge, England, 1966).

[15] I. T. Todorov, Analytic Properties of Feynman Diagrams in Quantum Field Theory (Pergamon, New York, 1971).

[16] H. H. Asatrian, H. M. Asatrian, C. Greub, and M. Walker, Phys. Lett. B 507, 162 (2001).

[17] P. Ball and R. Zwicky, Phys. Lett. B 642, 478 (2006).

[18] P. Ball, G. W. Jones, and R. Zwicky, Phys. Rev. D 75, 054004 (2007).

[19] L. Maiani and M. Testa, Phys. Lett. B 245, 585 (1990).

[20] A. Khodjamirian, Nucl. Phys. B605, 558 (2001).

[21] A. V. Smilga and M. A. Shifman, Yad. Fiz. 37, 1613 (1983) [Sov. J. Nucl. Phys. 37, 958 (1983)]; B. L. Ioffe and A. V. Smilga, Nucl. Phys. B216, 373 (1983).

[22] R. Mertig, M. Bohm, and A. Denner, Comput. Phys. Commun. 64, 345 (1991).

[23] G. Passarino and M. J. G. Veltman, Nucl. Phys. B160, 151 (1979).

[24] E. Bagan, P. Ball, and V. M. Braun, Phys. Lett. B 417, 154 (1998).

[25] T. M. Aliev and V. L. Eletsky, Yad. Fiz. 38, 1537 (1983) [Sov. J. Nucl. Phys. 38, 936 (1983)].

[26] J. Lyon and R. Zwicky, Report No. Edinburgh 05/13 (to be published).

[27] J. Charles, A. Le Yaouanc, L. Oliver, O. Pene, and J. C. Raynal, Phys. Rev. D 60, 014001 (1999).
[28] P. Ball and R. Zwicky, Phys. Rev. D 71, 014029 (2005).

[29] Y.-L. Wu, M. Zhong, and Y.-B. Zuo, Int. J. Mod. Phys. A 21, 6125 (2006).

[30] V. L. Chernyak and I. R. Zhitnitsky, Nucl. Phys. B345, 137 (1990).

[31] M. Beneke, G. Buchalla, M. Neubert, and C. T. Sachrajda, Nucl. Phys. B591, 313 (2000).

[32] T. Muta, Foundations of Quantum Chromodynamics, Lecture Notes in Physics Vol. 57 (World Scientific, Singapore, 1998).

[33] V. M. Braun, A. Khodjamirian, and M. Maul, Phys. Rev. D 61, 073004 (2000).

[34] E. Bagan, P. Ball, V. M. Braun, and H. G. Dosch, Phys. Lett. B 278, 457 (1992).

[35] T. Becher and M. Neubert, Eur. Phys. J. C 71, 1665 (2011).

[36] J.-Y. Chiu, A. Jain, D. Neill, and I.Z. Rothstein, J. High Energy Phys. 05 (2012) 084.

[37] S. J. Lee, M. Neubert, and G. Paz, Phys. Rev. D 75, 114005 (2007).

[38] Y. Amhis et al. (Heavy Flavor Averaging Group), arXiv:1207.1158.

[39] P. Ball, V. M. Braun, and N. Kivel, Nucl. Phys. B649, 263 (2003).

[40] C. Allton et al. (RBC-UKQCD Collaboration), Phys. Rev. D 78, 114509 (2008).

[41] R. Arthur, P. A. Boyle, D. Brommel, M. A. Donnellan, J. M. Flynn, A. Juttner, T. D. Rae, and C. T. C. Sachrajda, Phys. Rev. D 83, 074505 (2011).

[42] P. Ball and V. M. Braun, Phys. Rev. D 54, 2182 (1996).

[43] P. Ball and G. W. Jones, J. High Energy Phys. 03 (2007) 069.

[44] P. Ball and R. Zwicky, Phys. Lett. B 633, 289 (2006).

[45] P. Ball and R. Zwicky, J. High Energy Phys. 04 (2006) 046.

[46] M. Jung, X.-Q. Li, and A. Pich, J. High Energy Phys. 10 (2012) 063.

[47] C. Fronsdal, K. Mahanthappa, and R. E. Norton, Phys. Rev. 127, 1847 (1962).

[48] T. Hahn and M. Perez-Victoria, Comput. Phys. Commun. 118, 153 (1999).

[49] S. Mandelstam, Phys. Rev. Lett. 4, 84 (1960).

[50] G. Källén and A. S. Wightman, Mat. Fys. Skr. Dan. Vid. Selsk. 1, 1 (1958).

[51] B. Anderson, Commun. Math. Phys. 25, 283 (1972). 